Research Article

\title{
A New Paradigm to Design a Class of Combined Ternary Subdivision Schemes
}

\author{
Humaira Mustanira Tariq ${ }^{(1)},{ }^{1}$ Rabia Hameed ${ }^{(D)},{ }^{2}$ and Ghulam Mustafa ${ }^{1}{ }^{1}$ \\ ${ }^{1}$ Department of Mathematics, The Islamia University of Bahawalpur, Bahawalpur 63100, Pakistan \\ ${ }^{2}$ Department of Mathematics, The Government Sadiq College Women University Bahawalpur, Bahawalpur 63100, Pakistan \\ Correspondence should be addressed to Ghulam Mustafa; ghulam.mustafa@iub.edu.pk
}

Received 23 November 2020; Revised 8 January 2021; Accepted 2 February 2021; Published 16 February 2021

Academic Editor: Jia-Bao Liu

Copyright (c) 2021 Humaira Mustanira Tariq et al. This is an open access article distributed under the Creative Commons Attribution License, which permits unrestricted use, distribution, and reproduction in any medium, provided the original work is properly cited.

\begin{abstract}
Subdivision schemes play a vital role in curve modeling. The curves produced by the class of $(2 n+2)$-point ternary scheme (Deslauriers and Dubuc (1989)) interpolate the given data while the curves produced by a class of $(2 n+2)$-point ternary B-spline schemes approximate the given data. In this research, we merge these two classes to introduce a consolidated and unified class of combined subdivision schemes with two shape control parameters in order to grow versatility for overseeing valuable necessities. However, the proposed class of subdivision schemes gives optimal smoothness in the final shapes, yet we can increase its smoothness by using a proposed general formula in form of its Laurent polynomial. The theoretical analysis of the class of subdivision schemes is done by using various mathematical tools and using their coding in the Maple environment. The graphical analysis of the class of schemes is done in the Maple environment by writing the codes based on the recursive mathematical expressions of the class of subdivision schemes.
\end{abstract}

\section{Introduction}

Nowadays, subdivision schemes have got great importance in the field of geometric modeling. It is an innovation which creates smooth shapes. This technique produces a sequence of refined polygons which converge to a limiting shape. New points are added at each refinement level in order to get a smooth final shape. The number of points which are inserted at each refinement level is known as the arity of the curve subdivision scheme. Binary schemes insert two new points at each refinement level between every old consecutive pair of points of the previous refinement level [1]. Similarly, ternary subdivision schemes insert three new points at each refinement level between every old consecutive pair of points of the previous refinement level. Hence, ternary schemes smooth the given sketch in fewer subdivision steps as compared to the binary subdivision schemes. If we move the single point of the control polygon, then the shape of the curve changes over the specific region. This region is known as the support of the scheme. Subdivision schemes give us local control on the shapes. Schemes with small support give better local control on shapes as compared to the schemes with large support size. Ternary schemes give us small support as compared to the binary subdivision scheme and hence give better local control on the shapes. Because of these two main characteristics of ternary schemes, we consider them superior to the binary subdivision schemes.

Subdivision schemes can also produce the shapes which pass through the initial data. These types of subdivision schemes are known as interpolatory subdivision schemes. These schemes have been introduced by [2-6]. The other types of subdivision schemes produce shapes which do not pass through the initial data. This type of subdivision schemes is known as approximating subdivision schemes and was introduced by [7-10]. The analysis of both types of schemes is done by $[11,12]$. In [13-16], the subdivision schemes are introduced with parameters which can produce both the interpolatory and approximating shapes. The combined behaviour of these combined subdivision schemes got more attention in curve modeling. 
In this paper, we present a class of ternary combined schemes which is obtained from the well-known classes of interpolatory and approximating subdivision schemes. We unify interpolatory and approximating classes of subdivision schemes into a single class of combined schemes by applying certain mathematical operations on their refinement rules. Consequently, we get a class of $(2 n+2)$-point ternary combined subdivision schemes. Two shape parameters are also inserted in the refinement rules to control the appearance of the limiting shapes. A sketch of our construction procedure is shown in Figure 1.

The remaining layout of the paper is as follows: in Section 2, basic definitions, notations, and established tools are introduced. In Section 3, we construct a parametric class of $(2 n+2)$-point ternary relaxed subdivision schemes. This class depends on two parameters. In Section 4, we discuss several features of this class. In Section 5, we present the geometrical influence of the schemes on the shapes. The conclusion of this research is presented in Section 6.

\section{Preliminaries}

In this section, we review some fundamental definitions and known realities about subdivision schemes, which structure the basis of the remainder of this paper. If $q^{k}=\left\{q_{i}^{k}\right\}_{i \in \mathbb{Z}}$ and $q^{k+1}=\left\{q_{i}^{k+1}\right\}_{i \in \mathbb{Z}}$ are two polygons at $k$ th and $(k+1)$ the level, then the ternary subdivision scheme $(\mathrm{SS})_{a}$ which produces $q^{k+1}$ from $q^{k}$ is defined as

$$
\left((\mathrm{SS})_{a} q^{k}\right)_{i}=q_{i}^{k+1}=\sum_{j \in \mathbb{Z}} a_{i-3 j} q_{j}^{k}, \quad i \in \mathbb{Z},
$$

where the set $a=\left\{a_{i}: i \in \mathbb{Z}\right\}$ of coefficients is called the mask of the subdivision scheme.

For the ternary subdivision scheme $(\mathrm{SS})_{a}$, a point at $(k+1)$-th level is calculated by

$$
\left((\mathrm{SS})_{a} q^{k}\right)_{3 i+l}=q_{3 i+l}^{k+1}=\sum_{\gamma \in \mathbb{Z}} a_{3 \gamma+l} q_{i-\gamma}^{k}, \quad i \in \mathbb{Z}, 0 \leq l \leq 2 .
$$

A necessary condition of the subdivision scheme for uniform convergence is that

$$
\sum_{j \in \mathbb{Z}} a_{3 j}=\sum_{j \in \mathbb{Z}} a_{3 j+1}=\sum_{j \in \mathbb{Z}} a_{3 j+2}=1 .
$$

In order to analyze the characteristics of the subdivision scheme, the $z$-transform of the mask is

$$
a(z)=\sum_{i \in \mathbb{Z}} a_{i} z^{i}, \quad z \in \mathbb{C} \backslash\{0\},
$$

which is also known as the Laurent polynomial of the scheme.

Definition 1. A subdivision scheme is uniformly convergent if, for any initial data $q^{0}=\left\{q_{i}^{0}: i \in \mathbb{Z}\right\}$, there exists a continuous function $f$, such that for any closed interval $I \subset \mathbb{R}$ that satisfies

$$
\lim _{k \rightarrow \infty} \sup _{j \in 3^{k} I}\left|f\left(\frac{j}{3^{k}}\right)-q_{j}^{k}\right|=0,
$$

where $f=S^{\infty} q^{0}$.

Definition 2. If we rewrite the Laurent polynomial of (SS) ${ }_{a}$ as $a(z)=\left(1+z+z^{2} / 3\right)^{k} \delta(z)$, where $\delta(z)$ has no factor of the form $\left(1+z+z^{2}\right)$ and $\bar{m}_{0}, \ldots, \bar{m}_{l}$ are nonzero coefficients of $\delta(z)$. Let $F_{0}, F_{1}, \ldots, F_{l}$ be $l \times l$ matrices with the elements given by $\left(F_{\hbar}\right)_{i, j}=\bar{m}_{\ell+i-3 j+\hbar}$, where $\hbar=0,1, \ldots, l$ while $i, j=1, \ldots, l$. Hölders regularity/continuity is defined as $r=k-\log _{n}(\mu)$, whereas the parameter $\mu$ is known as a joint spectral radius of $F_{0}, F_{1}, \ldots, F_{l}$. It is bounded below by spectral radii and from above by the norm of matrices $F_{0}, F_{1}, \ldots, F_{l}$. If lower and upper bounds coincide, an explicit formula for the joint spectral radius is obtained:

$$
\begin{aligned}
& \left.\left.\max \left\{\rho\left(F_{0}\right), \rho\left(F_{1}\right), \ldots, \rho\left(F_{l}\right)\right),\right)\right\} \\
& \leq \mu \leq \max \left\{\left\|F_{0}\right\|_{\infty},\left\|F_{1}\right\|_{\infty}, \ldots,\left\|F_{l}\right\|_{\infty}\right\} .
\end{aligned}
$$

Theorem 1 (see [17]). If $S_{a}$ is the subdivision scheme with Laurent polynomial a $(z)$, then $S_{a}$ is said to be $C^{j}$-continuous if the subdivision scheme $(1 / 3) S_{n}$ is contractive. That is,

$$
\left\|\left(\frac{1}{3} S_{n}\right)^{L}\right\|_{\infty}=\frac{1}{3^{L}} \max \left\{\sum_{j \in \mathbb{Z}}\left|a_{i+3_{j}^{L}}^{[n, L]}\right| ; i=0,1,2, \ldots, 3^{L}-1\right\}<1,
$$

where

$$
\begin{aligned}
a^{[n, L]} & =\prod_{j=1}^{L} a\left(z^{3^{j-1}}\right), \\
a^{(n)}(z) & =\left(\frac{3 z^{2}}{1+z+z^{2}}\right) a^{(n-1)}(z) .
\end{aligned}
$$

\section{Formulation of the Class of Combined Schemes}

Here, we construct and unify the generalized form of the refinement rules of the class of interpolating and approximating schemes. The construction of the class of combined subdivision schemes is based on two classes of subdivision schemes. One of which is the class of $(2 n+2)$-point ternary interpolatory subdivision schemes (CETISS), which is constructed by the Lagrange interpolating polynomial, and the other one is the class of $(2 n+2)$-point relaxed ternary approximating subdivision schemes (CETASS), which is constructed by the B-spline basis function. We merge these classes of schemes in a specific manner such that we get a single class of $(2 n+2)$-point combined schemes from them.

3.1. Mathematical Representation of the Construction Procedure. In this subsection, we present the stepwise procedure to construct the class of combined ternary 


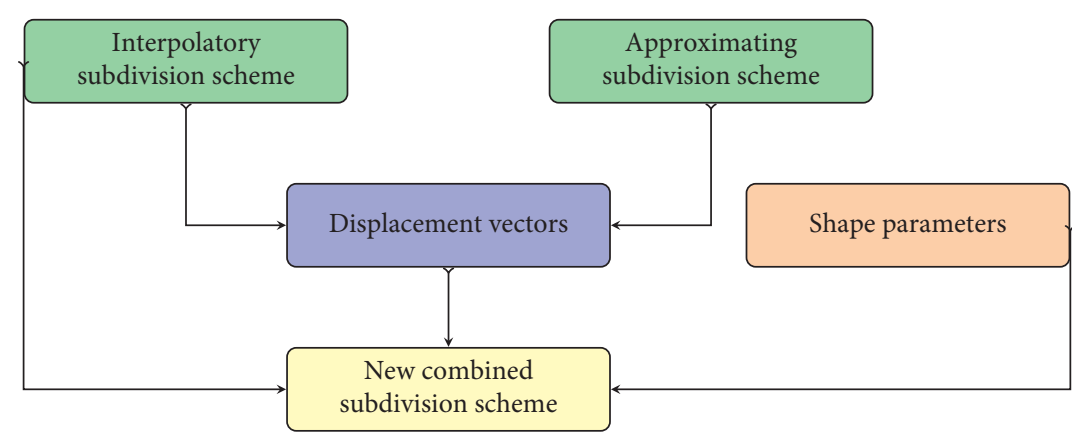

FIGURE 1: Flowchart for the construction of combined subdivision schemes.

subdivision schemes. The construction procedure of the class of combined subdivision schemes is given below.

Step 1. We define the generalized form of the refinement rules of the CETISS; that is, if we have a shape which is obtained by joining the $2 \mathrm{D}$ points $q_{i, 2 n+2}^{k}: i \in \mathbb{Z}$, then, to refine this shape by CETISS, we use the following refinement rules:

$$
\left\{\begin{array}{l}
L_{3 i, 2 n+2}^{k+1}=q_{i, 2 n+2}^{k}, \\
L_{3 i+1,2 n+2}^{k+1}=\frac{1}{3^{3 n+1}} \sum_{j=-n}^{n+1} \frac{(-1)^{j}(n-j+2)}{(1-3 j)}\left(\begin{array}{c}
3 n+2 \\
n
\end{array}\right)\left(\begin{array}{c}
2 n+2 \\
n+j
\end{array}\right) q_{i+j, 2 n+2}^{k}, \\
L_{3 i+2,2 n+2}^{k+2}=\frac{1}{3^{3 n+1}} \sum_{j=-n-1}^{n+1} \frac{(-1)^{j+1}(n+j+1)}{(3 j-2)}\left(\begin{array}{c}
3 n+2 \\
n
\end{array}\right)\left(\begin{array}{c}
2 n+2 \\
n+j+1
\end{array}\right) q_{i+j, 2 n+2}^{k},
\end{array}\right.
$$

and, hence, we get a refined shape by joining the $2 \mathrm{D}$ refined points $L_{i, 2 n+2}^{k+1}: i \in \mathbb{Z}$, where $\left(\begin{array}{l}n \\ r\end{array}\right)=(n ! / r !(n-r) !)$.

Step 2. By Pitolli [18], ternary B-spline schemes of degree- $M$ can be written as

$$
B_{\delta, M}^{k+1}=\frac{1}{3^{M}} \sum_{\eta=\{(\delta+1 / 2)\rfloor}^{\delta}\left(\begin{array}{c}
M+1 \\
\eta
\end{array}\right)\left(\begin{array}{c}
\eta \\
\delta-\eta
\end{array}\right), \quad 0 \leq \delta \leq 2(M+1) .
$$

$$
\left\{\begin{array}{l}
S_{3 i, 2 n+2}^{k+1}=\frac{1}{3^{3 m+1}} \sum_{\eta=0}^{2 m} \sum_{\gamma=\lfloor(3 \eta+3 / 2)\rfloor}^{3 \eta+2}\left(\begin{array}{c}
3 m+2 \\
\gamma
\end{array}\right)\left(\begin{array}{c}
\gamma \\
3 \eta+2-\gamma
\end{array}\right) q_{i+\eta-m, 2 n+2}^{k}, \\
S_{3 i+1,2 n+2}^{k+1}=\frac{1}{3^{3 m+1}} \sum_{\eta=0}^{2 m+1} \sum_{\gamma=\lfloor(3 \eta+2 / 2)\rfloor}^{3 \eta+2}\left(\begin{array}{c}
3 m+2 \\
\gamma
\end{array}\right)\left(\begin{array}{c}
\gamma \\
3 \eta+1-\gamma
\end{array}\right) q_{i+\eta-m, 2 n+2}^{k}, \\
S_{3 i+2,2 n+2}^{k+1}=\frac{1}{3^{3 m+1}} \sum_{\eta=0}^{2 m+1} \sum_{\gamma=\lfloor(3 \eta+1 / 2)\rfloor}^{3 \eta+2}\left(\begin{array}{c}
3 m+2 \\
\gamma
\end{array}\right)\left(\begin{array}{c}
\gamma \\
3 \eta-\gamma
\end{array}\right) q_{i+\eta-m, 2 n+2}^{k},
\end{array}\right.
$$


and thus we get a refined shape by joining the $2 \mathrm{D}$ refined points $S_{i, 2 n+2}^{k+1}: i \in \mathbb{Z}$.

Step 3. Now we calculate the displacement vectors from the refined points of CETISS, that is, $L_{3 i+\alpha, 2 n+2}^{k+1}: \alpha=0,1,2$ to the refined points of CETASS, that is, $S_{3 i+\alpha, 2 n+2}^{k+1}: \alpha=0,1,2$. We denote these displacement vectors by $\Delta_{3 i+\alpha, 2 n+2}^{k+1}$ where $\alpha=0,1,2$, respectively. Hence the displacement vectors are

$$
\Delta_{3 i+\alpha, 2 n+2}^{k+1}=L_{3 i+\alpha, 2 n+2}^{k+1}-S_{3 i+\alpha, 2 n+2}^{k+1},
$$

where $\alpha=0,1,2$.

Step 4. Now we find the new refinement point $q_{3 i+\alpha, 2 n+2}^{k+1}: \alpha=$ $0,1,2$ by using the displacement vectors $\Delta_{3 i+\alpha, 2 n+2}^{k+1}: \alpha=0,1,2$ and the refined points $L_{3 i+\alpha, 2 n+2}^{k+1}: \alpha=0,1,2$, respectively. We control the position of the refinement points $q_{3 i+\alpha, 2 n+2}^{k+1}: \alpha=$ $0,1,2$ by controlling the direction and size of these displacement vectors. The shape parameters $\mu$ and $\nu$ control the direction and size of the displacements vectors. The proposed refined points are given below:

$$
\left\{\begin{array}{l}
q_{3 i, 2 n+2}^{k+1}=L_{3 i, 2 n+2}^{k+1}-(\mu+\nu) \Delta_{3 i, 2 n+2}^{k+1} \\
q_{3 i+1,2 n+2}^{k+1}=L_{3 i+1,2 n+2}^{k+1}-\mu \Delta_{3 i+1,2 n+2}^{k+1}-v \Delta_{3 i+2,2 n+2}^{k+1} \\
q_{3 i+2,2 n+2}^{k+1}=L_{3 i+2,2 n+2}^{k+1}-v \Delta_{3 i+1,2 n+2}^{k+1}-\mu \Delta_{3 i+2,2 n+2}^{k+1}
\end{array}\right.
$$

Step 5. Hence we get the refinement rules $q_{3 i+\alpha, 2 n+2}^{k+1}: \alpha=$ $0,1,2$ by using (9), (12), and (13). That is,

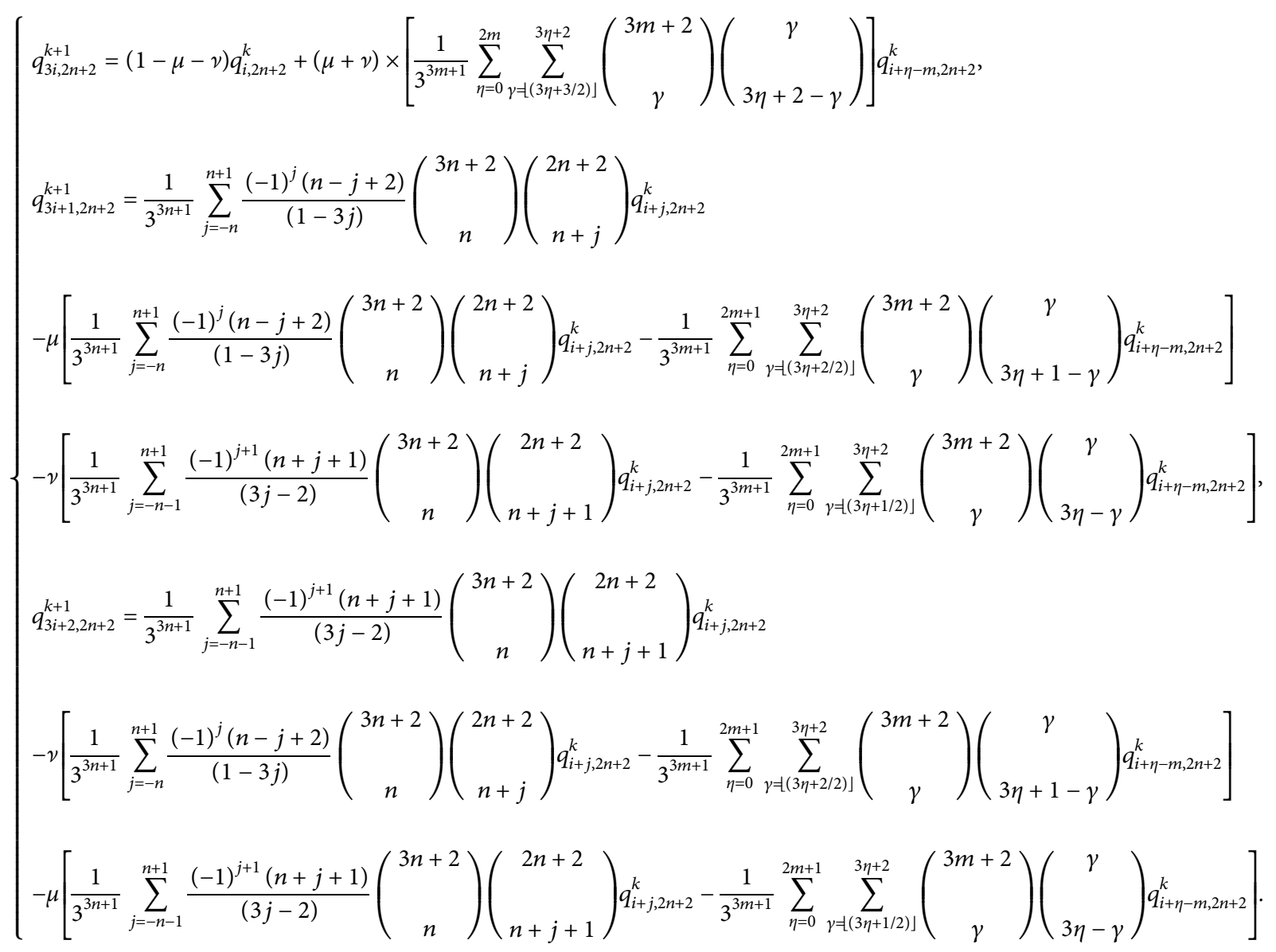

where $\lfloor\cdot\rfloor$ denotes the floor function. In this article, we denote the class of combined subdivision schemes (14) by $(\mathrm{CSS})_{a_{2 n+2}}$.
3.2. The Graphical Clarification of the Parameters and the Refinement Rules. In this subsection, we graphically explain the construction of the class of subdivision schemes. For this, 
we restrict the value of $n$ to be 1 . Let $q_{i+\alpha, 4}^{k}=q_{i+\alpha}^{k}: \alpha=-1,0,1,2$ be the control points of the polygon $q^{k} q^{k}$; then, for $n=1$, the class of subdivision schemes (9) gives the following 4-point ternary interpolatory subdivision scheme:

$$
\left\{\begin{array}{l}
L_{3 i}^{k+1}=L_{3 i, 4}^{k+1}=q_{i}^{k}, \\
L_{3 i+1}^{k+1}=L_{3 i+1,4}^{k+1}=\frac{-5}{81} q_{i-1}^{k}+\frac{60}{81} q_{i}^{k}+\frac{30}{81} q_{i+1}^{k}-\frac{4}{81} q_{i+2}^{k}, \\
L_{3 i+2}^{k+1}=L_{3 i+2,4}^{k+1}=\frac{-4}{81} q_{i-1}^{k}+\frac{30}{81} q_{i}^{k}+\frac{60}{81} q_{i+1}^{k}-\frac{5}{81} q_{i+2}^{k} .
\end{array}\right.
$$

Similarly, for $n=1$, the class of scheme (11) gives the following 4-point relaxed approximating subdivision scheme:

$$
\left\{\begin{array}{l}
S_{3 i}^{k+1}=S_{3 i, 4}^{k+1}=\frac{5}{27} q_{i-1}^{k}+\frac{17}{27} q_{i}^{k}+\frac{5}{27} q_{i+1}^{k} \\
S_{3 i+1}^{k+1}=S_{3 i+1,4}^{k+1}=\frac{5}{81} q_{i-1}^{k}+\frac{5}{9} q_{i}^{k}+\frac{10}{27} q_{i+1}^{k}+\frac{1}{81} q_{i+2}^{k} \\
S_{3 i+2}^{k+1}=S_{3 i+2,4}^{k+1}=\frac{1}{81} q_{i-1}^{k}+\frac{10}{27} q_{i}^{k}+\frac{5}{9} q_{i+1}^{k}+\frac{5}{81} q_{i+2}^{k}
\end{array}\right.
$$

Now, we find the displacement vectors from the refined points defined in (15) to the refined points defined in (16); thus, we have

$$
\begin{aligned}
& \Delta_{3 i}^{k+1}=\Delta_{3 i, 4}^{k+1}=L_{3 i}^{k+1}-S_{3 i}^{k+1}=\frac{10}{27} q_{i}^{k}-\frac{5}{27} q_{i-1}^{k}-\frac{5}{27} q_{i+1}^{k} \\
& \Delta_{3 i+1}^{k+1}=\Delta_{3 i+1,4}^{k+1}=L_{3 i+1}^{k+1}-S_{3 i+1}^{k+1}=-\frac{10}{81} q_{i-1}^{k}+\frac{5}{27} q_{i}^{k}-\frac{5}{81} q_{i+2}^{k} \\
& \Delta_{3 i+2}^{k+1}=\Delta_{3 i+2,4}^{k+1}=L_{3 i+2}^{k+1}-S_{3 i+2}^{k+1}=-\frac{5}{81} q_{i-1}^{k}+\frac{5}{27} q_{i+1}^{k}-\frac{10}{81} q_{i+2}^{k}
\end{aligned}
$$

Now, we find the refined points by applying operations on vectors $-(\mu+\nu) \Delta_{3 i}^{k+1},-\mu \Delta_{3 i+1}^{k+1},-\nu \Delta_{3 i+2}^{k+1}$ and refined points $L_{3 i+\alpha}^{k+1}: \alpha=0,1,2$, where $\mu$ and $\nu$ are real numbers. Hence, we get

$$
\left\{\begin{array}{l}
q_{3 i}^{k+1}=q_{3 i, 4}^{k+1}=L_{3 i}^{k+1}-(\mu+\nu) \Delta_{3 i}^{k+1}, \\
q_{3 i+1}^{k+1}=q_{3 i+1,4}^{k+1}=L_{3 i+1}^{k+1}-\mu \Delta_{3 i+1}^{k+1}-\nu \Delta_{3 i+2}^{k+1}, \\
q_{3 i+2}^{k+1}=q_{3 i+2,4}^{k+1}=L_{3 i+2}^{k+1}-\nu \Delta_{3 i+1}^{k+1}-\mu \Delta_{3 i+2}^{k+1} .
\end{array}\right.
$$

By simplifying (18), we get

$$
\left\{\begin{array}{l}
q_{3 i}^{k+1}=\left(\frac{5 \mu}{27}+\frac{5 \nu}{27}\right) q_{i-1}^{k}+\left(1-\frac{10 \mu}{27}-\frac{10 \nu}{27}\right) q_{i}^{k}+\left(\frac{5 \mu}{27}+\frac{5 \nu}{27}\right) q_{i+1}^{k} \\
q_{3 i+1}^{k+1}=\left(\frac{-5}{81}+\frac{5 \nu}{81}+\frac{10 \mu}{81}\right) q_{i-1}^{k}+\left(\frac{20}{27}-\frac{5 \mu}{27}\right) q_{i}^{k}+\left(\frac{10}{27}-\frac{5 \nu}{27}\right) q_{i+1}^{k}+\left(\frac{-4}{81}+\frac{5 \mu}{81}+\frac{10 \nu}{81}\right) q_{i+2}^{k} \\
q_{3 i+2}^{k+1}=\left(\frac{-4}{81}+\frac{5 \mu}{81}+\frac{10 \nu}{81}\right) q_{i-1}^{k}+\left(\frac{10}{27}-\frac{5 \nu}{27}\right) q_{i}^{k}+\left(\frac{20}{27}-\frac{5 \mu}{27}\right) q_{i+1}^{k}+\left(\frac{-5}{81}+\frac{5 v}{81}+\frac{10 \mu}{81}\right) q_{i+2}^{k}
\end{array}\right.
$$

Scheme (19) is the first member of the class of combined subdivision schemes, that is, 4-point combined subdivision scheme. The graphical sketch of the refined points of (19) is shown in Figure 2. In this figure, green bullets show the points $q_{i}^{k}: i \in \mathbb{Z}$, green lines show the polygon $q^{k}$, red squares show the refined points $L_{3 i+\alpha}^{k+1}: \alpha \in \mathbb{R}$, red lines show the refined polygon $L^{k+1}$, blue squares show the refined points $S_{3 i+\alpha}^{k+1}: \alpha \in \mathbb{R}$, blue lines show the refined polygon $S^{k+1}$, black arrows show the displacement vectors, and black solid circles show the refined points $q_{i, 2 n+2}^{k+1}=q_{i}^{k+1}: i \in \mathbb{Z}$.

Remark 1. When $\mu=\nu=0$, the first member of the class of combined subdivision schemes which is defined in (19) reduces to the first member of the CETISS. And when $\mu=1$ and $\nu=0$, the first member of the class of combined subdivision schemes which is defined in (19) reduces to the first member of the CETASS.

\section{Analysis of the Class of Combined Subdivision Schemes by Using Mathematical Tools}

In this section, we present the characteristics of the class of combined subdivision schemes. We use the mathematical tools to analyze the class of schemes.

4.1. Affine Combination of Control Points. A linear combination in which the sum of coefficients is equal to one is called the affine combination. The necessary condition for the convergence of a subdivision scheme is that each refinement rule of it must be the affine combination of the control points in the previous subdivision step. To check this property for $(\mathrm{CSS})_{a_{2 n+2}}$, we prove the following theorem.

Theorem 2. Each refined point of the $(C S S)_{a_{2 n+2}}$ at $(k+1)$-th subdivision step which is defined in (14) is the affine combination of control points of $k$-th subdivision step. 


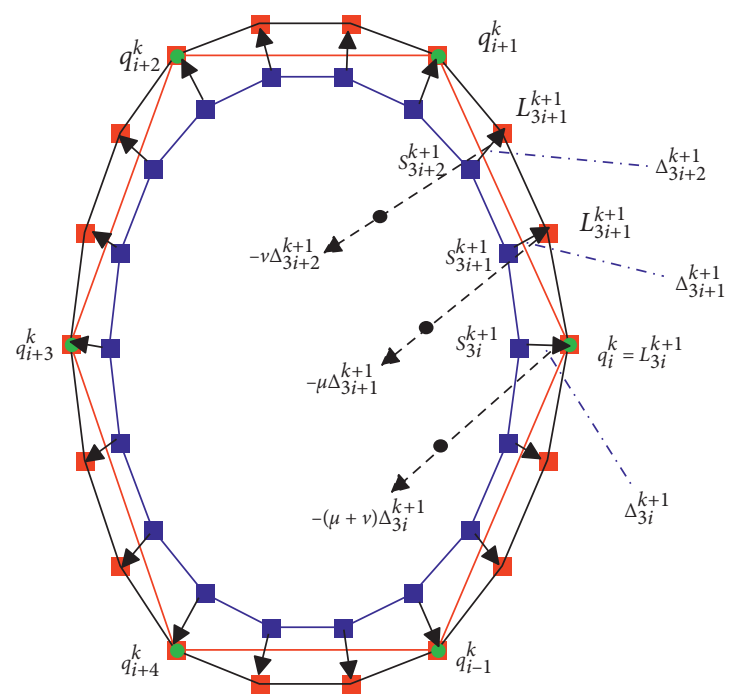

FIGURE 2: Graphical sketch of the construction of the refinement points of scheme (19).

Proof. To prove this theorem, we have to prove that the mask $\left\{a_{i, 2 n+2}: i \in \mathbb{Z}\right\}$ of $(\mathrm{CSS})_{a_{2 n+2}}$ satisfies the relation $\sum_{i \in \mathbb{Z}} a_{3 i+\alpha, 2 n+2}=1$, where $\alpha=0,1,2$.

Let us simplify the expressions:

$$
\left\{\begin{array}{l}
\sum_{\eta=0}^{2 m} \sum_{\gamma=(3 \eta+3 / 2)\rfloor}^{3 \eta+2}\left(\begin{array}{c}
3 m+2 \\
\gamma
\end{array}\right)\left(\begin{array}{c}
\gamma \\
3 \eta+2-\gamma
\end{array}\right)=3^{3 m+1}, \\
\sum_{\eta=0}^{2 m+1} \sum_{\gamma=(3 \eta+2 / 2)\rfloor}^{3 \eta+2}\left(\begin{array}{c}
3 m+2 \\
\gamma
\end{array}\right)\left(\begin{array}{c}
\gamma \\
3 \eta+1-\gamma
\end{array}\right)=3^{3 m+1}, \\
\sum_{\eta=0}^{2 m+1} \sum_{\gamma=(3 \eta+1 / 2)\rfloor}^{3 \eta+2}\left(\begin{array}{c}
3 m+2 \\
\gamma
\end{array}\right)\left(\begin{array}{c}
\gamma \\
3 \eta-\gamma
\end{array}\right)=3^{3 m+1} .
\end{array}\right.
$$

We also calculate

$$
\left\{\begin{array}{l}
\sum_{j=-n}^{n+1} \frac{(-1)^{j}(n-j+2)}{(1-3 j)}\left(\begin{array}{c}
3 n+2 \\
n
\end{array}\right)\left(\begin{array}{c}
2 n+2 \\
n+j
\end{array}\right)=3^{3 n+1}, \\
\sum_{j=-n-1}^{n+1} \frac{(-1)^{j+1}(n+j+1)}{(3 j-2)}\left(\begin{array}{c}
3 n+2 \\
n
\end{array}\right)\left(\begin{array}{c}
2 n+2 \\
n+j+1
\end{array}\right)=3^{3 n+1} .
\end{array}\right.
$$

Hence, to prove that each of the refined points $q_{3 i, 2 n+2}^{k+1}$, $q_{3 i+1,2 n+2}^{k+1}$, and $q_{3 i+2,2 n+2}^{k+1}$ is the affine combinations of $\left\{q_{i}^{k}: i \in \mathbb{Z}\right\}$, we use (20) and (21). Thus,

$$
\left\{\begin{array}{l}
\sum_{i \in \mathbb{Z}} a_{3 i, 2 n+2}=(1-\mu-\nu)+(\mu+\nu)\left[\frac{1}{3^{3 m+1}} 3^{3 m+1}\right]=1, \\
\sum_{i \in \mathbb{Z}} a_{3 i+1,2 n+2}=\frac{1}{3^{3 n+1}} 3^{3 n+1}-\mu\left[\frac{1}{3^{3 n+1}} 3^{3 n+1}-\frac{1}{3^{3 m+1}} 3^{3 m+1}\right]-\nu\left[\frac{1}{3^{3 n+1}} 3^{3 n+1}-\frac{1}{3^{3 m+1}} 3^{3 m+1}\right]=1, \\
\sum_{i \in \mathbb{Z}} a_{3 i+2,2 n+2}=\frac{1}{3^{3 n+1}} 3^{3 n+1}-\nu\left[\frac{1}{3^{3 n+1}} 3^{3 n+1}-\frac{1}{3^{3 m+1}} 3^{3 m+1}\right]-\mu\left[\frac{1}{3^{3 n+1}} 3^{3 n+1}-\frac{1}{3^{3 m+1}} 3^{3 m+1}\right]=1,
\end{array}\right.
$$

which completes the proof.

4.2. Support of the Class of Combined Schemes. In this subsection, we present the support size of the class of combined schemes. The support of a subdivision scheme represents how far one vertex is affected by its neighboring points. Its size represents the local support property of the subdivision curve.
Theorem 3. The support of the class of subdivision schemes $(C S S)_{a_{2 n+2}}$ is $[-((3 n+2) / 2),((3 n+2) / 2)]$.

Proof. Let $\left\{q_{i, 2 n+2}^{0}=q_{i}^{0} \in \mathbb{R}\right\}_{i \in \mathbb{Z}}$ be the initial data such that $q_{0}^{0}=1$ and $q_{\alpha}^{0} \neq 0$ for $\alpha \in \mathbb{Z} \backslash\{0\}$; that is,

$$
q_{\alpha, 2 n+2}^{0}= \begin{cases}1, & \text { for } \alpha=0, \\ 0, & \text { otherwise. }\end{cases}
$$


In order to calculate the support size of $(\mathrm{CSS})_{a_{2 n+2}}$, we have to calculate the distance between two corresponding subscripts belonging to the leftmost vertex and rightmost vertex after $k$-steps of subdivision.
If we operate on initial data $\left\{q_{i, 2 n+2}^{0}=q_{i}^{0} \in \mathbb{R}\right\}_{i \in \mathbb{Z}}$ by $(\mathrm{CSS})_{a_{2 n+2}}$ one time, then we get

$$
q_{\alpha, 2 n+2}^{1}= \begin{cases}\neq 0, & \text { for } \alpha=-3^{0}(3 n+2),-3^{0}(3 n+2)+1, \ldots, 3^{0}(3 n+2)-1,3^{0}(3 n+2) \\ 0, & \text { otherwise. }\end{cases}
$$

If we operate on the data $\left\{q_{i, 2 n+2}^{1} \in \mathbb{R}\right\}_{i \in \mathbb{Z}}$ which is defined in (24) by (CSS) $a_{a_{2 n+2}}$ one time, that is, we operate on

initial data $\left\{q_{i, 2 n+2}^{0}=q_{i}^{0} \in \mathbb{R}\right\}_{i \in \mathbb{Z}}$ by (CSS $)_{a_{2 n+2}}$ two times, then we get

$$
q_{\alpha, 2 n+2}^{2}= \begin{cases}\neq 0, & \text { for } \alpha=-\sum_{j=0}^{1} 3^{j}(3 n+2),-\sum_{j=0}^{1} 3^{j}(3 n+2)+1, \ldots, \sum_{j=0}^{1} 3^{j}(3 n+2)-1, \sum_{j=0}^{1} 3^{j}(3 n+2), \\ 0, & \text { otherwise. }\end{cases}
$$

Similarly, if we operate on initial data $\left\{q_{i, 2 n+2}^{0}=q_{i}^{0} \in \mathbb{R}\right\}_{i \in \mathbb{Z}} \quad$ which is defined in (23) by

(CSS) $a_{2 n+2} k$-times, then we get

$$
q_{\alpha, 2 n+2}^{k}= \begin{cases}\neq 0, & \text { for } \alpha=-\sum_{j=0}^{k-1} 3^{j}(3 n+2),-\sum_{j=0}^{k-1} 3^{j}(3 n+2)+1, \ldots, \sum_{j=0}^{k-1} 3^{j}(3 n+2)-1, \sum_{j=0}^{k-1} 3^{j}(3 n+2), \\ 0, & \text { otherwise. }\end{cases}
$$

Hence, after $k$-steps of subdivision, the leftmost nonzero

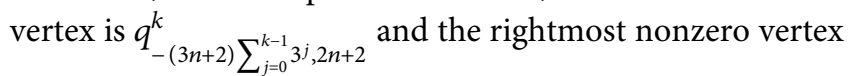
is $q_{(3 n+2) \sum_{j=0}^{k-1} 3^{j}, 2 n+2}^{k}$. Since $k$-times, the subdivision process is applied and, at each step, we have relabeled the subscripts of the vertices; thus, $\quad q_{\left(i / 3^{k}\right), 2 n+2}^{k}=q_{\left(i / 3^{k-1}\right), 2 n+}$ $2^{k-1}=q_{\left(i / 3^{k-2}\right), 2 n+2}^{k-2}=\cdots=q_{(i / 3), 2 n+2}^{1}=q_{i, 2 n+2}^{0}$. Hence, after $k$-steps, the distance between leftmost and rightmost nonzero vertices is equal to the support of (CSS $)_{a_{2 n+2}}$; that is,

$$
\begin{aligned}
\text { support } & =\left[\frac{(3 n+2)\left(1+3+3^{2}+\cdots+3^{k-1}\right)}{3^{k}}-\frac{-(3 n+2)\left(1+3+3^{2}+\cdots+3^{k-1}\right)}{3^{k}}\right] \\
& =2(3 n+2)\left(\frac{1}{3}+\frac{1}{3^{2}}+\cdots+\frac{1}{3^{k}}\right) \\
& =2(3 n+2)\left(\frac{(1 / 3)}{1-(1 / 3)}\right) \\
& =(3 n+2) .
\end{aligned}
$$

Since the class of subdivision schemes is symmetric, then obviously the support region is [(- $(3 n+2) / 2),((3 n+2) / 2)]$.
4.3. Generation Degree. One of the necessary conditions for generating $C^{n}$ - continuous limit curves is the order of polynomial generation of the subdivision scheme. A ternary 
subdivision scheme can generate polynomials up to degree $d$ if and only if its Laurent polynomial can be written in the following form:

$$
a(z)=\left(\frac{1+z+z^{2}}{3}\right)^{d+1} \varphi(z), \quad z \in \mathbb{C} \backslash\{0\} .
$$

Here, $\varphi(z)$ is the Laurent polynomial with $\varphi(1)=3$ and it does not have the factor $\left(1+z+z^{2}\right)$.

Theorem 4. The class of combined schemes $(C S S)_{a_{2 n+2}}$ generates polynomials of degree up to 2 for all $\mu$ and $\nu$.

Proof. The Laurent polynomial of (CSS $)_{a_{2 n+2}}$ is

$$
a_{2 n+2}(z)=\left(\frac{1+z+z^{2}}{3}\right)^{3} b_{2 n+2}(z),
$$

where $b_{2 n+2}(z)$ is a Laurent polynomial obtained by $b_{2 n+2}(z)=\left(3 a_{2 n+2}(z) / 1+z+z^{2}\right)$. Hence, the degree of polynomial generation of $(\mathrm{CSS})_{a_{2 n+2}}$ is 2 .

4.4. Properties of the 4-Point Subdivision Scheme (19). From Theorem 2, the subdivision scheme which is defined in (14) and denoted by (CSS) $a_{4}$ satisfies the necessary conditions for convergence. Moreover, the Laurent polynomial of $(\mathrm{CSS})_{a_{4}}$ is

$$
a_{4}(z)=\frac{1}{81 z^{5}}\left(1+z+z^{2}\right)^{3}\left[(10 \nu-4+5 \mu) z^{4}+(-25 \nu+7-5 \mu) z^{3}+(30 \nu+3) z^{2}+(-25 \nu+7-5 \mu) z+(10 \nu-4+5 \mu)\right]
$$

By using Laurent polynomial of $(\mathrm{CSS})_{a_{4}}$, we prove the following theorem.

Theorem 5. Let $\left\{q_{i}^{0}\right\}_{i \in \mathbb{Z}}$ be the initial data; then, the refinement rules defined in (19) of $(\mathrm{CSS})_{a_{4}}$ produce the $\mathrm{C}^{2}$ continuous shapes if $\nu \in((-2 / 5),(1 / 5))$ and its corresponding $\mu \in((1 / 5)-(7 v / 2), 2-(7 v / 2))$.

Proof. To prove that $(\mathrm{CSS})_{a_{4}}$ produces the $C^{2}$-continuous shapes, first, we have to prove that this scheme produces
$C^{0}$-continuous and $C^{1}$-continuous shapes in the given intervals of $\mu$ and $\nu$.

Thus, by using (30), we calculate

$$
a_{4}^{(1)}(z)=\left(\frac{3 z^{2}}{1+z+z^{2}}\right) a_{4}^{(0)}(z) .
$$

Hence, from the above equation, we have

$$
\begin{aligned}
a_{4}^{(1)}(z) & =\frac{1}{27 z^{2}}\left[\begin{array}{c}
(-4+5 \mu+10 \nu) z^{5}+(-1+5 \mu-5 \nu) z^{4}+ \\
(10 \nu+5 \mu+5) z^{3}+(26-10 \mu-20 \nu) z^{2}+(29+10 \nu-10 \mu) z^{1}+(26-10 \mu-20 \nu) z^{0}
\end{array}\right] \\
& =\sum_{j=-2}^{3} a_{j, 4} z^{j}
\end{aligned}
$$

where the subdivision scheme corresponding to the Laurent polynomial $a_{4}^{(1)}(z)$ is denoted by $(\mathrm{CSS})_{a_{4}^{(1)}}$; then, by Theorem 1 , we have

$$
\left\|\left(\frac{1}{3}(\mathrm{CSS})_{a_{4}^{(1)}}\right)^{1}\right\|_{\infty}=\frac{1}{3} \max \left\{\sum_{j \in \mathbb{Z}}\left|a_{i+3 j, 4}^{[1,1]}\right| ; i=0,1,2\right\} .
$$

This implies that

$$
\left\|\left(\frac{1}{3}(\mathrm{CSS}) a_{4}^{(1)}\right)^{1}\right\|_{\infty}=\frac{1}{3} \max \left\{\sum_{j \in \mathbb{Z}}\left|a_{3 j, 4}^{[1,1]}\right|, \sum_{j \in \mathbb{Z}}\left|a_{1+3 j, 4}^{[1,1]}\right|, \sum_{j \in \mathbb{Z}}\left|a_{2+3 j, 4}^{[1,1]}\right|\right\} .
$$

By substituting values, we get

$$
\begin{aligned}
& \left\|\left(\frac{1}{3}(\mathrm{CSS})_{a_{4}^{(1)}}\right)^{1}\right\|_{\infty}=\max \frac{1}{81} \\
& \{|-4+5 \mu+10 \nu|+|26-10 \mu-20 \nu|+|10 \nu+5 \mu+5|,|-1+5 \mu-5 \nu|+|29+10 \nu-10 \mu|+|-1+5 \mu-5 \nu|\} .
\end{aligned}
$$


Hence, $\quad\left\|\left((1 / 3)(\mathrm{CSS})_{a_{4}^{(1)}}\right)^{1}\right\|_{\infty}<1 \quad$ for $\mu+2 \nu \in((-14 / 5),(53 / 10))$ and $\mu-v \in((5 / 2),(28 / 5))$.

This implies that (CSS) $a_{4}^{(1)}$ is contractive and the scheme $(\mathrm{CSS})_{a_{4}}$ produces $C^{0}$-continuous shapes.

Now, we again calculate

$$
a_{4}^{(2)}(z)=\left(\frac{3 z^{2}}{1+z+z^{2}}\right) a_{4}^{(1)}(z)
$$

which implies

$$
\begin{aligned}
& a_{4}^{(1)}(z)= \frac{1}{9 z^{2}} \\
& {\left[(10 \nu-4+5 \mu) z^{5}+(3-15 v) z^{4}+(6+15 v) z^{3}\right.} \\
& \quad+(-10 \mu+17-20 v) z^{2}+(6+15 v) z^{1} \\
&\left.\quad+(3-15 v) z^{0}+(10 v-4+5 \mu) z^{-1}\right]
\end{aligned}
$$

where the subdivision scheme corresponding to the Laurent polynomial $a_{4}^{(2)}(z)$ is denoted by $(\mathrm{CSS})_{a_{4}^{(2)}}$; then, by Theorem 1 , we have

$$
\begin{aligned}
& \left\|\left(\frac{1}{3}(\mathrm{CSS})_{a_{4}^{(2)}}\right)^{1}\right\|_{\infty}=\max \frac{1}{27} . \\
& \{|-4+5 \mu+10 \nu|+|17-10 \mu-20 \nu| \\
& \quad+|10 \nu+5 \mu-4|,|3-15 \nu|+|6+15 \nu|\} .
\end{aligned}
$$

Thus, $\left\|\left((1 / 3)(\mathrm{CSS})_{a^{(2)}}\right)^{1}\right\|_{\infty}<1$ for $v \in(-1,(4 / 5))$ and $\mu \in((-1 / 10)-2 v,-2 v+(13 / 5))$.

Therefore, $(\mathrm{CSS})_{a_{4}^{(2)}}$ is contractive and hence $(\mathrm{CSS})_{a_{4}}$ produces $C^{1}$-continuous shapes.
Now, we find the following Laurent polynomial by using $a_{4}^{(3)}(z)=\left(3 z^{2} / 1+z+z^{2}\right) a_{4}^{(2)}(z)$. Thus, we have

$$
\begin{aligned}
& a_{4}^{(3)}(z)= \frac{1}{3} \\
& \quad\left[(10 \nu-4+5 \mu) z^{5}+(-25 v+7-5 \mu) z^{4}+(30 \nu+3) z^{3}\right. \\
&\left.\quad+(-25 v+7-5 \mu) z^{2}+(10 v-4+5 \mu) z\right] .
\end{aligned}
$$

Let $(\mathrm{CSS})_{a_{4}^{(3)}}$ be the subdivision scheme corresponding to the Laurent polynomial $a_{4}^{(3)}(z)$; then, by Theorem 1 , we get

$$
\begin{aligned}
& \left\|\left(\frac{1}{3}(\mathrm{CSS})_{a_{4}^{(3)}}\right)^{1}\right\|_{\infty}=\max \frac{1}{9}\{|-4+5 \mu+10 \nu| \\
& \quad+|7-5 \mu-25 \nu|,|3+30 \nu|\} .
\end{aligned}
$$

Thus, $\left\|\left((1 / 3)(\mathrm{CSS}) a^{(3)}\right)^{1}\right\|_{\infty}<1$ for $v \in((-2 / 5),(1 / 5))$ and $\mu \in((1 / 5)-(7 v / 2), 2-(7 v / 2))$.

This implies that (CSS) $a_{4}^{(3)}$ is contractive and hence $(\mathrm{CSS})_{a_{4}}$ is $C^{2}$-continuous. This completes the proof.

If we put $v=0$ in the Laurent polynomial (30), then it gives one more factor of $\left(1+z+z^{2}\right)$. Hence, the following corollary is proved.

Corollary 1. If $\nu=0$ and $\mu \in((4 / 5),(6 / 5))$, then $(C S S)_{a_{4}}$ produces $C^{3}$-continuous shapes.

Corollary 2. The continuity of (CSS $)_{a_{4}}$ can be increased if we use the refinement rules corresponding to the following Laurent polynomial which is derived from the Laurent polynomial of (30):

$$
\begin{aligned}
& \alpha(z)=\frac{1}{3^{n+2}}\left[C_{0}^{n}\left\{C_{0}^{n}(10 v-4+5 \mu)\right\}+C_{0}^{n}\left\{C_{0}^{n}(-15 v+3)+C_{1}^{n}(10 v-4+5 \mu)\right\} z\right. \\
& +\left(C_{0}^{n}\left\{C_{0}^{n}(15 v+6)+C_{1}^{n}(-15 v+3)+C_{2}^{n}(10 v-4+5 \mu)\right\}+C_{1}^{n}\left\{C_{0}^{n-1}(10 \nu-4+5 \mu)\right\}\right) z^{2} \\
& +\left(C_{0}^{n}\left\{C_{0}^{n}(-20 \nu-10 \mu+17)+C_{1}^{n}(15 v+6)+C_{2}^{n}(-15 v+3)+C_{3}^{n}(10 \nu-4+5 \mu)\right\}\right. \\
& \left.+C_{1}^{n}\left\{C_{0}^{n-1}(-15 v+3)+C_{1}^{n-1}(10 \nu-4+5 \mu)\right\}\right) z^{3} \\
& +\left(C_{0}^{n}\left\{C_{0}^{n}(15 v+6)+C_{1}^{n}(-20 v-10 \mu+17)+C_{2}^{n}(15 v+6)+C_{3}^{n}(-15 v+3)\right\}\right. \\
& \left.+C_{1}^{n}\left\{C_{0}^{n-1}(15 v+6)+C_{1}^{n-1}(-15 v+3)+C_{2}^{n-1}(10 v-4+5 \mu)\right\}+C_{2}^{n}\left\{C_{0}^{n-2}(10 v-4+5 \mu)\right\}\right) z^{4} \\
& +\cdots++\left(C_{n}^{n-2}\left\{C_{2}^{2}(10 \nu-4+5 \mu)\right\}\right) z^{2 n+4} \\
& +\left(C_{n-1}^{n}\{(10 v-4+5 \mu)+(-15 v+3)\}+C_{n}^{n}(-15 v+3)\right) z^{2 n+5} \\
& \left.+\left(C_{n}^{n}(10 v-4+5 \mu)\right) z^{2 n+6}\right]
\end{aligned}
$$

where $\mu$ and $\nu$ are shape parameters and $n=1,2, \ldots$..
Remark 2. If we put $n=2, n=3$, and $n=4$ in (41), we get the Laurent polynomials of 3 -point, 4-point, and 5-point 
combined subdivision schemes which produce continuous shapes up to $C^{2}, C^{3}$, and $C^{4}$ smoothness.

Theorem 3 gives the following result.

Corollary 3. The support of $(C S S)_{a_{4}}$ is $[-(5 / 2),(5 / 2)]$.

Now, here we use the method proposed by Rioul [19] to find out the Höolders regularity of $(\mathrm{CSS})_{a_{4}}$.

Theorem 6. If we choose $\mu=1$ and $\nu=(-1 / 5)$, then the lower and upper bound of Hölder regularity of $(C S S)_{a_{4}}$ is 2.1079 and 2.369 , respectively.

Proof. From (30), we have

$$
a_{4}(z)=\left(\frac{1+z+z^{2}}{3}\right)^{3} z^{-5} \sum_{j=0}^{4} m_{j} z^{j},
$$

where $m_{0}=((10 v-4+5 \mu) / 3), \quad m_{1}=((-25 v+7-5 \mu) / 3)$, $m_{2}=((30 \nu+3) / 3), \quad m_{3}=((-25 \nu+7-5 \mu) / 3)$, $m_{4}=((10 v-4+5 \mu) / 3), \quad k=3, \quad \ell=4, \quad n=3, \quad$ and $\hbar=0,1,2,3,4 . F_{0}, F_{1}, F_{2}, F_{3}$, and $F_{4}$ are matrices given by

$$
\left(F_{\hbar}\right)_{i, j}=m_{\ell+i-3 j+\hbar}
$$

Since $\left(F_{0}\right)_{i, j}=m_{4+i-3 j},\left(F_{1}\right)_{i, j}=m_{5+}$ $i-3 j,\left(F_{2}\right)_{i, j}=m_{6+i-3 j},\left(F_{3}\right)_{i, j}=m_{7+i-3 j},\left(F_{4}\right)_{i, j}=m_{8+i-3 j}$, where $i, j=1,2,3,4$, thus, we have

$$
\begin{aligned}
& F_{0}=\left(\begin{array}{cccc}
m_{2} & 0 & 0 & 0 \\
m_{3} & m_{0} & 0 & 0 \\
m_{4} & m_{1} & 0 & 0 \\
0 & m_{2} & 0 & 0
\end{array}\right), \\
& F_{1}=\left(\begin{array}{cccc}
m_{3} & m_{0} & 0 & 0 \\
m_{4} & m_{1} & 0 & 0 \\
0 & m_{2} & 0 & 0 \\
0 & m_{3} & m_{0} & 0
\end{array}\right) \text {, } \\
& F_{2}=\left(\begin{array}{cccc}
m_{4} & m_{1} & 0 & 0 \\
0 & m_{2} & 0 & 0 \\
0 & m_{3} & m_{0} & 0 \\
0 & m_{4} & m_{1} & 0
\end{array}\right) \text {, } \\
& F_{3}=\left(\begin{array}{cccc}
0 & m_{2} & 0 & 0 \\
0 & m_{3} & m_{0} & 0 \\
0 & m_{4} & m_{1} & 0 \\
0 & 0 & m_{2} & 0
\end{array}\right) \text {, } \\
& F_{4}=\left(\begin{array}{cccc}
0 & m_{3} & m_{0} & 0 \\
0 & m_{4} & m_{1} & 0 \\
0 & 0 & m_{2} & 0 \\
0 & 0 & m_{3} & m_{0}
\end{array}\right) .
\end{aligned}
$$

Let $\rho\left(F_{j}\right): j=0,1,2,3,4$ be the joint spectral radii of matrices $F_{0}, F_{1}, F_{2}, F_{3}$, and $F_{4}$, respectively; then, we have

$$
\begin{aligned}
& \max \left\{\rho\left(F_{0}\right), \rho\left(F_{1}\right), \rho\left(F_{2}\right), \rho\left(F_{3}\right), \rho\left(F_{4}\right)\right\} \\
& \leq \rho \leq \max \left\{\left\|F_{0}\right\|_{\infty},\left\|F_{1}\right\|_{\infty},\left\|F_{2}\right\|_{\infty},\left\|F_{3}\right\|_{\infty},\left\|F_{4}\right\|_{\infty}\right\} .
\end{aligned}
$$

The eigenvalues of $F_{0}, F_{1}, F_{2}, F_{3}$, and $F_{4}$ are required to calculate their joint spectral radii; therefore, $\rho\left(F_{0}\right)=\rho\left(F_{2}\right)=\rho\left(F_{3}\right)=\rho\left(F_{4}\right)=, \max \left\{\left|m_{o}\right|,\left|m_{2}\right|\right\}$, $\rho\left(F_{1}\right)=, \max \left\{\left|m_{3}+m_{0}\right|,\left|m_{1}+m_{4}\right|\right\}$.

The infinity norm of $F_{0}, F_{1}, F_{2}, F_{3}$, and $F_{4}$ is

$$
\left\|F_{j}\right\|_{\infty}=\max \left\{\left|m_{2}\right|,\left|m_{3}+m_{0}\right|,\left|m_{4}+m_{1}\right|\right\} \text {, }
$$

where $j=0,1,2,3,4$.

$$
\begin{aligned}
& \max \left\{\left|m_{0}\right|,\left|m_{2}\right|,\left|m_{3}+m_{0}\right|,\left|m_{1}-m_{4}\right|\right\} \\
& \leq \rho \leq \max \left\{\left|m_{2}\right|,\left|m_{3}+m_{0}\right|,\left|m_{4}+m_{1}\right|\right\} .
\end{aligned}
$$

If we use $\mu=1$ and $\nu=(-1 / 5)$ in (47), we get

$$
\max \{1,0.333,2,2.6667\} \leq \rho \leq \max \{1,2\} .
$$

Hence, the lower bound of Höölders exponent is

$$
r \geq 3-\log _{3}(2.6667)=2.10719 \text {. }
$$

The upper bound of Hölders exponent is

$$
r \leq 3-\log _{3}(2)=2.369 \text {. }
$$

This completes the proof.

Theorem 7. The limit stencil of $(\mathrm{CSS})_{a_{4}}$ which is defined in (18) is

$$
\left[\frac{5 \mu+5 \nu-3}{48}, \frac{27-5 \mu-5 \nu}{48}, \frac{27-5 \mu-5 \nu}{48}, \frac{5 \mu+5 \nu-3}{48}\right] .
$$

Proof. To find out the limit stencil of $(\mathrm{CSS})_{a_{4}}$, we substitute $i=-1$ and $i=0$ in the refinement rules (19) of $(\mathrm{CSS})_{a_{4}}$. Hence, we obtain the following refined points:

$$
\begin{aligned}
q_{-3}^{k+1}= & \left(\frac{5 \mu}{27}+\frac{5 \nu}{27}\right) q_{-2}^{k}+\left(1-\frac{10 \mu}{27}-\frac{10 \nu}{27}\right) q_{-1}^{k}+\left(\frac{5 \mu}{27}+\frac{5 \nu}{27}\right) q_{0}^{k} \\
q_{-2}^{k+1}= & \left(\frac{-5}{81}+\frac{5 \nu}{81}+\frac{10 \mu}{81}\right) q_{-2}^{k}+\left(\frac{20}{27}-\frac{5 \mu}{27}\right) q_{-1}^{k}+\left(\frac{10}{27}-\frac{5 \nu}{27}\right) q_{0}^{k} \\
& +\left(\frac{-4}{81}+\frac{5 \mu}{81}+\frac{10 \nu}{81}\right) q_{1}^{k}, \\
& +\left(\frac{-5}{81}+\frac{5 \nu}{81}+\frac{10 \mu}{81}\right) q_{1}^{k}, \\
q_{-1}^{k+1}= & \left(\frac{-4}{81}+\frac{5 \mu}{81}+\frac{10 \nu}{81}\right) q_{-2}^{k}+\left(\frac{10}{27}-\frac{5 \nu}{27}\right) q_{-1}^{k}+\left(\frac{20}{27}-\frac{5 \mu}{27}\right) q_{0}^{k} \\
q_{0}^{k+1}= & \left(\frac{5 \mu}{27}+\frac{5 \nu}{27}\right) q_{-1}^{k}+\left(1-\frac{10 \mu}{27}-\frac{10 \nu}{27}\right) q_{0}^{k}+\left(\frac{5 \mu}{27}+\frac{5 \nu}{27}\right) q_{1}^{k} .
\end{aligned}
$$


The matrix form of the above system of four linear equations is

$$
\left(\begin{array}{c}
q_{-3}^{k+1} \\
q_{-2}^{k+1} \\
q_{-1}^{k+1} \\
q_{0}^{k+1}
\end{array}\right)\left(\begin{array}{cccc}
\left(\frac{5 \mu}{27}+\frac{5 \nu}{27}\right) & \left(1-\frac{10 \mu}{27}-\frac{10 \nu}{27}\right) & \left(\frac{5 \mu}{27}+\frac{5 \nu}{27}\right) & 0 \\
\left(\frac{-5}{81}+\frac{5 \nu}{81}+\frac{10 \mu}{81}\right) & \left(\frac{20}{27}-\frac{5 \mu}{27}\right) & \left(\frac{10}{27}-\frac{5 \nu}{27}\right) & \left(\frac{-4}{81}+\frac{5 \mu}{81}+\frac{10 \nu}{81}\right) \\
\left(\frac{-4}{81}+\frac{5 \mu}{81}+\frac{10 \nu}{81}\right) & \left(\frac{10}{27}-\frac{5 \nu}{27}\right) & \left(\frac{20}{27}-\frac{5 \mu}{27}\right) & \left(\frac{-5}{81}+\frac{5 \nu}{81}+\frac{10 \mu}{81}\right) \\
0 & \left(\frac{5 \mu}{27}+\frac{5 \nu}{27}\right) & \left(1-\frac{10 \mu}{27}-\frac{10 \nu}{27}\right) & \left(\frac{5 \mu}{27}+\frac{5 \nu}{27}\right)
\end{array}\right)\left(\begin{array}{c}
q_{-3}^{k} \\
q_{-2}^{k} \\
q_{-1}^{k} \\
q_{0}^{k}
\end{array}\right) .
$$

Symbolically, it can be written as

$$
q^{k+1}=R_{1} q^{j}
$$

Hence, the local subdivision matrix of the 4-point scheme $(\mathrm{CSS})_{a_{4}}$ is

$$
R_{1}=\left(\begin{array}{cccc}
\left(\frac{5 \mu}{27}+\frac{5 \nu}{27}\right) & \left(1-\frac{10 \mu}{27}-\frac{10 \nu}{27}\right) & \left(\frac{5 \mu}{27}+\frac{5 \nu}{27}\right) & 0 \\
\left(\frac{-5}{81}+\frac{5 \nu}{81}+\frac{10 \mu}{81}\right) & \left(\frac{20}{27}-\frac{5 \mu}{27}\right) & \left(\frac{10}{27}-\frac{5 \nu}{27}\right) & \left(\frac{-4}{81}+\frac{5 \mu}{81}+\frac{10 \nu}{81}\right) \\
\left(\frac{-4}{81}+\frac{5 \mu}{81}+\frac{10 \nu}{81}\right) & \left(\frac{10}{27}-\frac{5 \nu}{27}\right) & \left(\frac{20}{27}-\frac{5 \mu}{27}\right) & \left(\frac{-5}{81}+\frac{5 \nu}{81}+\frac{10 \mu}{81}\right) \\
0 & \left(\frac{5 \mu}{27}+\frac{5 \nu}{27}\right) & \left(1-\frac{10 \mu}{27}-\frac{10 \nu}{27}\right) & \left(\frac{5 \mu}{27}+\frac{5 \nu}{27}\right)
\end{array}\right) .
$$


The matrix of the eigenvector corresponding to the eigenvalues is

$$
W=\left(\begin{array}{cccc}
1 & -1 & 1 & -1 \\
\frac{5 \mu+5 \nu-3}{5 \mu+5 \nu-27} & \frac{-1}{3} & 1 & \frac{5 \nu-5 \mu+1}{15 \mu+15 \nu-27} \\
\frac{5 \mu+5 \nu-3}{5 \mu+5 \nu-27} & \frac{1}{3} & 1 & \frac{-(5 \nu-5 \mu+1)}{15 \mu+15 \nu-27} \\
1 & 1 & 1 & 1
\end{array}\right) .
$$

By taking the inverse of the above matrix, we get

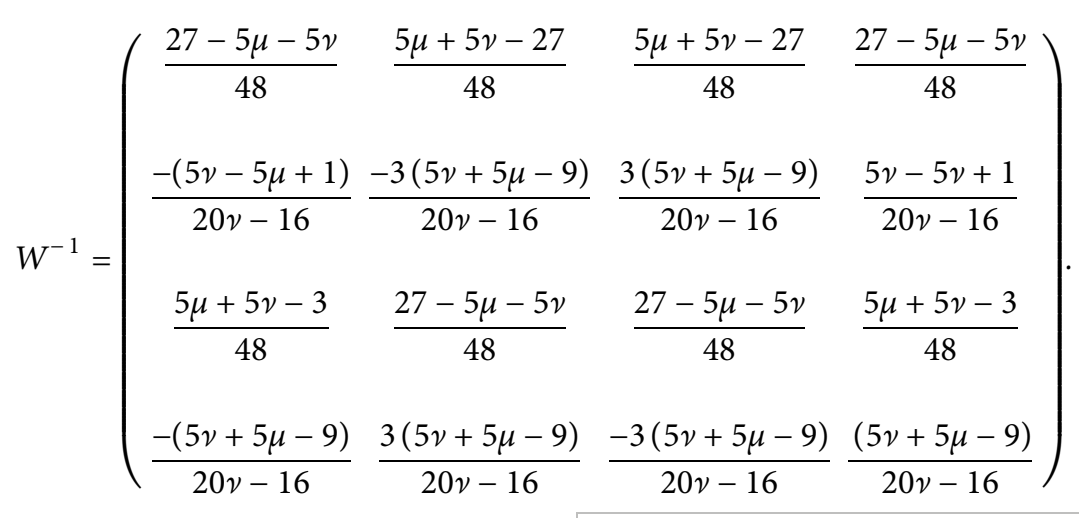

Let $\delta$ be the diagonal matrix of eigenvalues; then,

$$
\delta=\left(\begin{array}{cccc}
1 & 0 & 0 & 0 \\
0 & \frac{1}{3} & 0 & 0 \\
0 & 0 & \frac{1}{9} & 0 \\
0 & 0 & 0 & \frac{(10 v+1)}{27}
\end{array}\right) .
$$
have

Since $R_{1}=W \delta W^{-1}$, therefore $R_{1}^{k}=W \delta^{k} W^{-1}$. Also, we

$$
q^{k+1}=R_{1} q^{k}=R_{1}^{2} q^{k-1}=R_{1}^{3} q^{k-2}=\cdots=R_{1}^{k} q^{0},
$$

which implies that

$$
q^{k+1}=W \delta^{k} W^{-1} q^{0}
$$

Hence

$$
\lim _{k \rightarrow \infty} q^{k+1}=W\left(\lim _{k \longrightarrow \infty} \delta^{k}\right) W^{-1} q^{0}
$$

Thus, we have

$$
\left(\begin{array}{c}
q_{-3}^{\infty} \\
q_{-2}^{\infty} \\
q_{-1}^{\infty} \\
q_{0}^{\infty}
\end{array}\right)=\left(\begin{array}{llll}
\frac{5 \mu+5 \nu-3}{48} & \frac{27-5 \mu-5 \nu}{48} & \frac{27-5 \mu-5 \nu}{48} & \frac{5 \mu+5 \nu-3}{48} \\
\frac{5 \mu+5 \nu-3}{48} & \frac{27-5 \mu-5 \nu}{48} & \frac{27-5 \mu-5 \nu}{48} & \frac{5 \mu+5 \nu-3}{48} \\
\frac{5 \mu+5 \nu-3}{48} & \frac{27-5 \mu-5 \nu}{48} & \frac{27-5 \mu-5 \nu}{48} & \frac{5 \mu+5 \nu-3}{48} \\
\frac{5 \mu+5 \nu-3}{48} & \frac{27-5 \mu-5 \nu}{48} & \frac{27-5 \mu-5 \nu}{48} & \frac{5 \mu+5 \nu-3}{48}
\end{array}\right)\left(\begin{array}{c}
q_{-3}^{0} \\
q_{-2}^{0} \\
q_{-1}^{0} \\
q_{0}^{0}
\end{array}\right)
$$


Hence, we get the limit stencil of $(\mathrm{CSS})_{a_{4}}$, that is, $\left[\frac{5 \mu+5 \nu-3}{48}, \frac{27-5 \mu-5 \nu}{48}, \frac{27-5 \mu-5 \nu}{48}, \frac{5 \mu+5 \nu-3}{48}\right]$. (64)
4.5. Properties of the 6-Point Subdivision Scheme. If we put $n=2$ in (14), then we get the following 6-point combined subdivision scheme. We denote this scheme by $(\mathrm{CSS})_{a_{6}}$ :

Hence, it is proved.

$$
\left\{\begin{array}{l}
q_{3 i}^{k+1}=\left(\frac{4 \mu}{243}+\frac{4 v}{243}\right) q_{i-2}^{k}+\left(\frac{56 \mu}{243}+\frac{56 \nu}{243}\right) q_{i-1}^{k}+\left(1-\frac{40 \mu}{81}-\frac{40 \nu}{81}\right) q_{i}^{k}+\left(\frac{56 \mu}{243}+\frac{56 \nu}{243}\right) q_{i+1}^{k}+\left(\frac{4 \mu}{243}+\frac{4 v}{243}\right) q_{i+2}^{k} \\
q_{3 i+1}^{k+1}=\left(\frac{-16 \mu}{2187}-\frac{20 \nu}{2187}+\frac{8}{729}\right) q_{i-2}^{k}+\left(\frac{-70}{729}+\frac{280 \nu}{2187}+\frac{476 \mu}{2187}\right) q_{i-1}^{k}+\left(\frac{560}{729}-\frac{664 \mu}{2187}-\frac{56 \nu}{2187}\right) q_{i}^{k} \\
+\left(\frac{280}{729}-\frac{56 \mu}{2187}-\frac{664 \nu}{2187}\right) q_{i+1}^{k}+\left(\frac{-56}{729}+\frac{280 \mu}{2187}+\frac{476 \nu}{2187}\right) q_{i+2}^{k}+\left(\frac{7}{729}-\frac{20 \mu}{2187}-\frac{16 \nu}{2187}\right) q_{i+3}^{k} \\
q_{3 i+2}^{k+1}=\left(\frac{7}{729}-\frac{20 \mu}{2187}-\frac{16 \nu}{2187}\right) q_{i-2}^{k}+\left(\frac{-56}{729}+\frac{280 \mu}{2187}+\frac{476 v}{2187}\right) q_{i-1}^{k}+\left(\frac{280}{729}-\frac{56 \mu}{2187}-\frac{664 v}{2187}\right) q_{i}^{k} \\
+\left(\frac{560}{729}-\frac{664 \mu}{2187}-\frac{56 \nu}{2187}\right) q_{i+1}^{k}+\left(\frac{-70}{729}+\frac{280 \nu}{2187}+\frac{476 \mu}{2187}\right) q_{i+2}^{k}+\left(-\frac{16 \mu}{2187}-\frac{20 \nu}{2187}+\frac{8}{729}\right) q_{i+3}^{k} .
\end{array}\right.
$$

The Laurent polynomial of (CSS $)_{a_{6}}$ denoted by $a_{6}(z)=$ $a_{6}^{(0)}(z)$ is defined:

$$
\begin{aligned}
& a_{6}(z)= \frac{1}{2187 z^{8}}\left(1+z+z^{2}\right)^{3} \\
& {\left[(21-20 \mu-16 v) z^{10}+(-39+44 \mu+28 v) z^{9}+(24 \mu+48 v-9) z^{8}+(-54+276 v+84 \mu) z^{7}\right.} \\
&+(-108 \mu-936 v+153) z^{6}+(99+1200 \nu-48 \mu) \times z^{5}+(-108 \mu-936 \nu+153) z^{4}+(-54+276 v+84 \mu) z^{3} \\
&\left.+(24 \mu+48 v-9) z^{2}+(-39+44 \mu+28 v) z+(21-20 \mu-16 v)\right] .
\end{aligned}
$$

Theorem 8. Let $\left\{q_{i}^{0}\right\}_{i \in \mathbb{Z}}$ be the initial data; then, the refinement rules defined in (65) of $(\mathrm{CSS})_{a_{6}}$ produce the $\mathrm{C}^{2}$ continuous shapes if $v \in((-1 / 4),(1 / 8))$ and its corresponding $\mu \in((3 / 32)-(157 v / 32),(255 / 128)-(157 v / 32))$. It gives $C^{3}$-continuous shapes for $\nu=0$ and $\mu \in((93 / 128),(87 / 64))$.
It produces $C^{4}$-smooth shapes for $\nu=0$ and $\mu \in((3 / 5),(51 / 40))$. And it gives $C^{5}$-smooth shapes for $v=0$ and $\mu \in((57 / 62),(33 / 31))$.

Proof. By Theorem 1, we have 
$a_{6}^{(n)}(z)=\left(\frac{3 z^{2}}{1+z+z^{2}}\right) a_{6}^{(n-1)}(z), \quad i=1,2,3,4,5,6$

Hence,

$$
\begin{aligned}
& a_{6}^{(1)}(z)=\frac{1}{729}\left[(21-20 \mu-16 v) z^{8}+(3-4 v+4 \mu) z^{7}+(-24+56 v+52 \mu) z^{6}+(224 \mu+424 v-147) z^{5}\right. \\
& +(-39-200 \nu+200 \mu) z^{4}+(280 \nu+186+80 \mu) z^{3}+(-336 \mu+693-744 \nu) z^{2} \\
& +(801+408 v-408 \mu) z^{1}+(-336 \mu+693-744 v) z^{0}+(280 \nu+186+80 \mu) z^{-1}+ \\
& (-39-200 \nu+200 \mu) z^{-2}+(224 \mu+424 \nu-147) z^{-3}+(-24+56 \nu+52 \mu) z^{-4} \\
& \left.+(3-4 \nu+4 \mu) z^{5}+(21-20 \mu-16 \nu) z^{-6}\right] \\
& a_{6}^{(2)}(z)=\frac{1}{243}\left[(21-20 \mu-16 \nu) z^{8}+(12 \nu+24 \mu-18) z^{7}+(60 \nu+48 \mu-27) z^{6}\right. \\
& +(152 \mu-102+352 \nu) z^{5}+(90-612 \nu) z^{4}+(198-72 \mu+540 \nu) z^{3} \\
& +(405-264 \mu-672 \nu) z^{2}+(198-72 \mu+540 \nu) z^{1}+(90-612 \nu) z^{0} \\
& +(152 \mu-102+352 v) z^{-1}+(60 \nu+48 \mu-27) z^{-2}+(12 v+24 \mu-18) z^{-3} \\
& \left.+(21-20 \mu-16 v) z^{-4}\right] \\
& a_{6}^{(3)}(z)=\frac{1}{81 z^{2}}\left[(21-20 \mu-16 v) z^{10}+(-39+44 \mu+28 v) z^{9}+(24 \mu+48 v-9) z^{8}\right. \\
& +(-54+276 v+84 \mu) z^{7}+(-108 \mu-936 v+153) z^{6}+(99+1200 v-48 \mu) z \\
& +(-108 \mu-936 v+153) z^{4}+(-54+276 v+84 \mu) z^{3}+(24 \mu+48 v-9) z^{25} \\
& +(-39+44 \mu+28 v) z+(21-20 \mu-16 \nu)] .
\end{aligned}
$$

And when $v=0$, we get the following Laurent polynomials from (67):

$$
\begin{aligned}
& a_{6}^{(4)}(z)=\frac{1}{27}\left[\begin{array}{c}
(21-20 \mu) z^{8}+(-60+64 \mu) z^{7}+(-20 \mu+30) z^{6}+(40 \mu-24) z^{5} \\
+(147-128 \mu) z^{4}+(40 \mu-24) z^{3}+(-20 \mu+30) z^{2}+(-60+64 \mu) z+(21-20 \mu)
\end{array}\right], \\
& a_{6}^{(5)}(z)=\frac{1}{9}\left[\begin{array}{c}
(21-20 \mu) z^{6}+(-81+84 \mu) z^{5}+(-84 \mu+90) z^{4}+(40 \mu-33) z^{3} \\
+(-84 \mu+90) z^{2}+(-81+84 \mu) z^{1}+(21-20 \mu)
\end{array}\right], \\
& \alpha^{(6)}(z)=\left(\frac{3 z^{2}}{1+z+z^{2}}\right) \frac{1}{9}\left[\begin{array}{c}
(21-20 \mu) z^{6}+(-81+84 \mu) z^{5}+(-84 \mu+90) z^{4}+(40 \mu-33) z^{3} \\
+(-84 \mu+90) z^{2}+(-81+84 \mu) z^{1}+(21-20 \mu)
\end{array}\right] .
\end{aligned}
$$

Then, by using Theorem 1 and denoting the subdivision schemes corresponding to the Laurent polynomials $a_{6}^{(j)}(z): j=0,1,2,3,4,5,6$ by $(\mathrm{CSS})_{a_{6}^{(j)}}: j=0,1,2,3,4,5,6$, respectively, we get 


$$
\left\|\left(\frac{1}{3}(\mathrm{CSS})_{a_{6}^{(j)}}\right)^{1}\right\|_{\infty}=\frac{1}{3} \max \left\{\sum_{j \in \mathbb{Z}}\left|\alpha_{i+3 j}^{[j, 1]}\right| ; i=0,1,2\right\}, \quad j=0,1,2,3,4,5,6 .
$$

By solving $\left\|\left((1 / 3)(\mathrm{CSS})_{a_{6}^{(j)}}\right)^{1}\right\|_{\infty}<1 \quad$ for $j=0,1,2,3,4,5,6$, we get the required result.
Corollary 4. The order of smoothness of $(\mathrm{CSS})_{a_{6}}$ can be increased if we use the refinement rules corresponding to the following Laurent polynomial which is derived from the Laurent polynomial of (66):

$$
\begin{aligned}
& \alpha(z)=\frac{1}{3^{n+5}}\left[C_{0}^{n}\left\{C_{0}^{n}(21-16 v-20 \mu)\right\}+C_{0}^{n}\left\{C_{0}^{n}(-18+12 v+24 \mu)+C_{1}^{n}(21-16 \nu-20 \mu)\right\} z\right. \\
& +\left(C_{0}^{n}\left\{C_{0}^{n}(48 \mu+60 \nu-27)+C_{1}^{n}(-18+12 v+24 \mu)+C_{2}^{n}(21-16 \nu-20 \mu)\right\}+C_{1}^{n}\left\{C_{0}^{n-1}(21-16 \nu-20 \mu)\right\}\right) z^{2} \\
& +\left(C_{0}^{n}\left\{C_{0}^{n}(152 \mu+352 \nu-102)+C_{1}^{n} \times(48 \mu+60 \nu-27)+C_{2}^{n}(-18+12 \nu+24 \mu)+C_{3}^{n}(21-16 \nu-20 \mu)\right\}+\right. \\
& \left.C_{1}^{n} \times\left\{C_{0}^{n-1}(-18+12 \nu+24 \mu)+C_{1}^{n-1}(21-16 \nu-20 \mu)\right\}\right) z^{3} \\
& +\left(C_{0}^{n}\left\{C_{0}^{n}(-612 \nu+90)+C_{1}^{n}(152 \mu+352 \nu-102)+C_{2}^{n}(48 \mu+60 \nu-27)+C_{3}^{n}(-18+12 \nu+24 \mu)\right\}+C_{1}^{n} \times\right. \\
& \left.\left\{C_{0}^{n-1}(48 \mu+60 \nu-2)+C_{1}^{n-1}(-18+12 \nu+24 \mu)+C_{2}^{n-1}(21-16 \nu-20 \mu)\right\}+C_{2}^{n}\left\{C_{0}^{n-2}(21-16 \nu-20 \mu)\right\}\right) z^{4} \\
& \cdots+\left(C_{n-2}^{n}\left\{C_{2}^{2}(21-16 \nu-20 \mu)\right\}+C_{n-1}^{n}\left\{C_{1}^{1}(21-16 v-20 \mu)+C_{0}^{1}(-18+12 v+24 \mu)\right\}+C_{n}^{n}(48 \mu+60 v-27)\right) z^{2 n+10} \\
& +\left(C_{n-1}^{n} \times\{(21-16 v-20 \mu)\}+C_{n}^{n}(-18+12 v+24 \mu)\right) z^{2 n+11} \\
& \left.\left(C_{n}^{n}(21-16 v-20 \mu)\right) z^{2 n+12}\right]
\end{aligned}
$$

where $\mu$ and $\nu$ are shape parameters and $m \in \mathbb{N}$.

Theorem 9. The limit stencil of $(C S S)_{a_{6}}$ is

Theorem 3 gives the following result.

Corollary 5. The support of $(C S S)_{a_{6}}$ is $[-4,4]$.

$$
\begin{aligned}
& \xi_{1}=\frac{64\left(\mu^{3}+\mu^{2} \nu-\mu \nu^{2}-\nu^{3}\right)-580 \mu^{2}-930 \mu \nu+555 \mu+350 \nu^{2}+555 v}{756\left(20 \mu+145 v-6 \mu \nu-6 v^{2}+240\right)}, \\
& \xi_{2}=\frac{-\left(12 \mu^{3}+40 \mu^{2} \nu-317 \mu^{2}+44 \mu \nu^{2}+45 \mu+16 \nu^{3}-644 \nu^{2}+45 \nu-961 \mu \nu\right)}{42\left(20 \mu+145 \nu-6 \mu \nu-6 \nu^{2}+240\right)}, \\
& \xi 3=\frac{32 \mu^{3}+88 \mu^{2} \nu-1396 \mu^{2}+80 \mu \nu^{2}-2138 \mu \nu+6525 \mu+24 \nu^{3}-742 \nu^{2}+6525 \nu}{84\left(20 \mu+145 \nu-6 \mu \nu-6 \nu^{2}+240\right)}, \\
& \xi 4=\frac{-68\left(\mu^{3}+\mu^{2} \nu-\mu \nu^{2}-\nu^{3}\right)+3719 \mu^{2}+303 \mu \nu-25455 \mu-3416 \nu^{2}-1830 \nu+45360}{189\left(20 \mu+145 \nu-6 \mu \nu-6 \nu^{2}+240\right)} .
\end{aligned}
$$

Theorem 10. If we choose $\mu=1$ and $\nu=(-1 / 5)$, then the lower and upper bound of Höolder regularity of $(C S S)_{a_{6}}$ are 2.1302 and 2.455, respectively. 


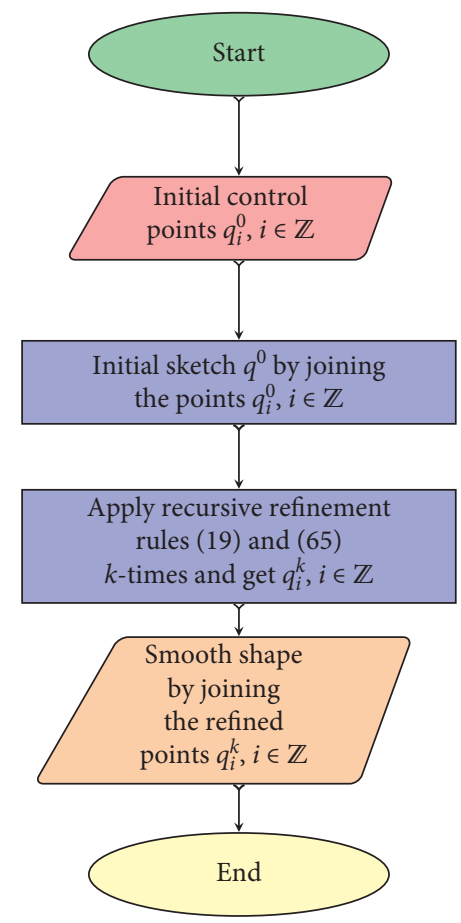

FIgURE 3: Flowchart to explain the geometric examples.
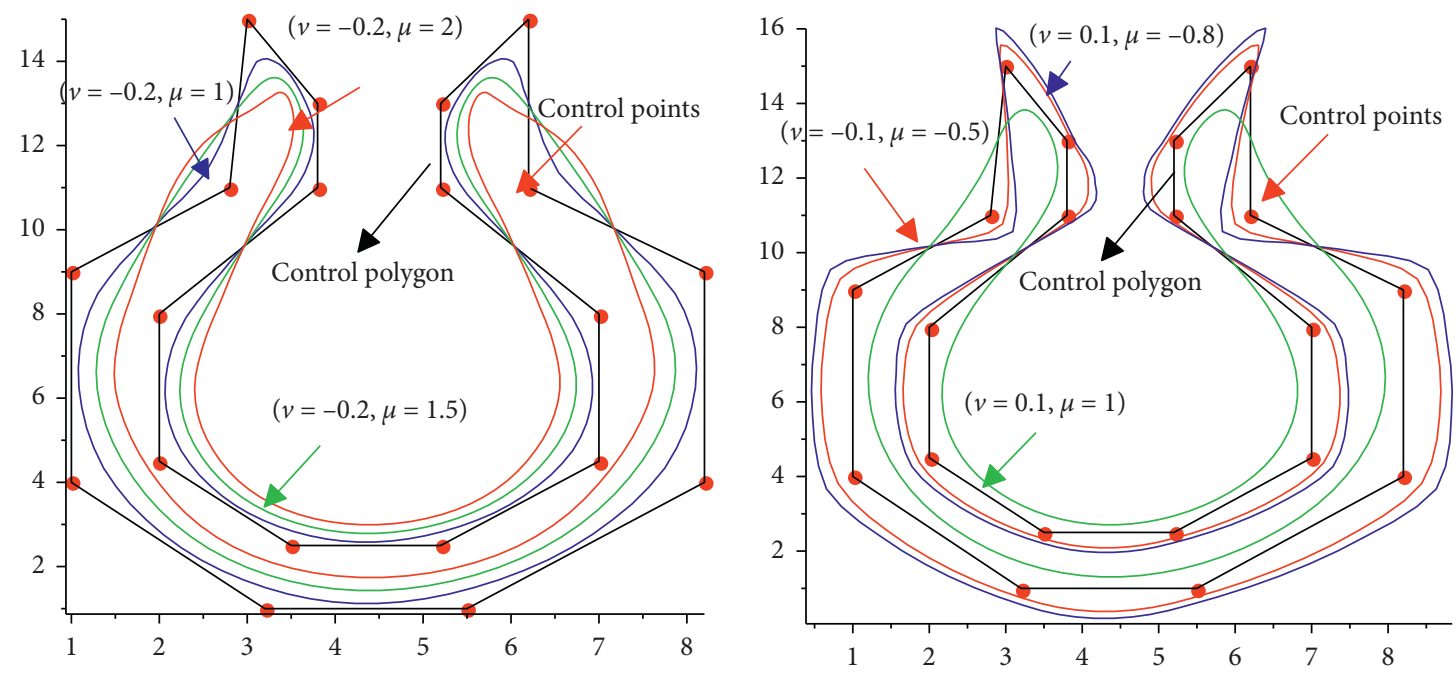

(a)

(b)

Figure 4: Continued. 


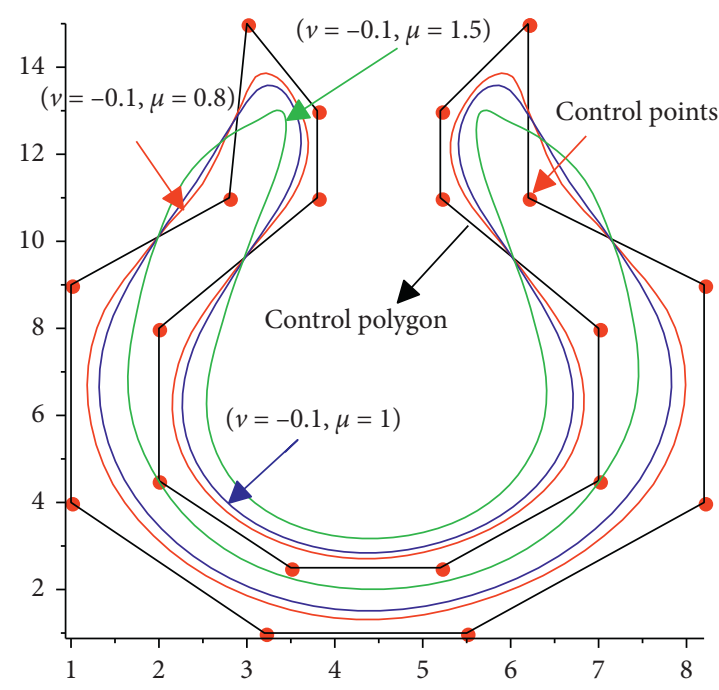

(c)

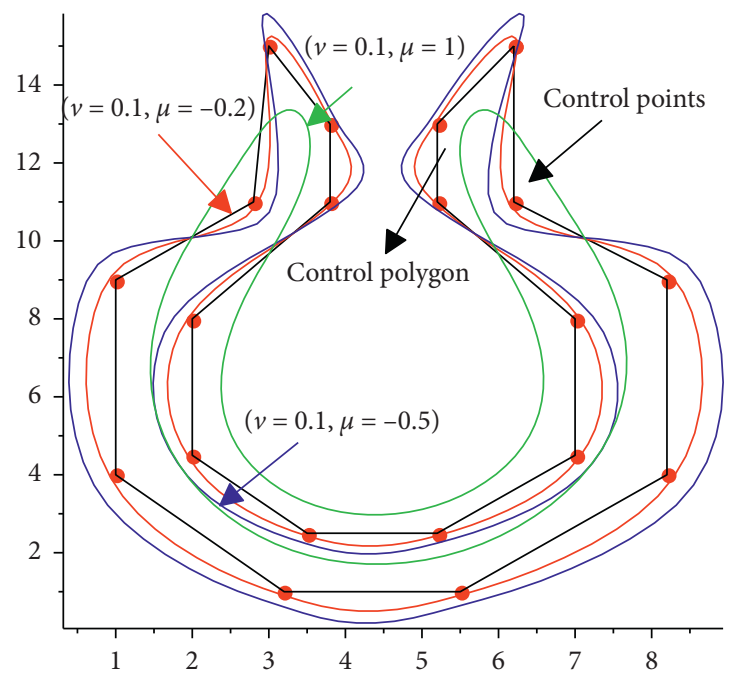

(d)

FIgURE 4: Coloured lines show the curves fitted by the combined subdivision schemes.

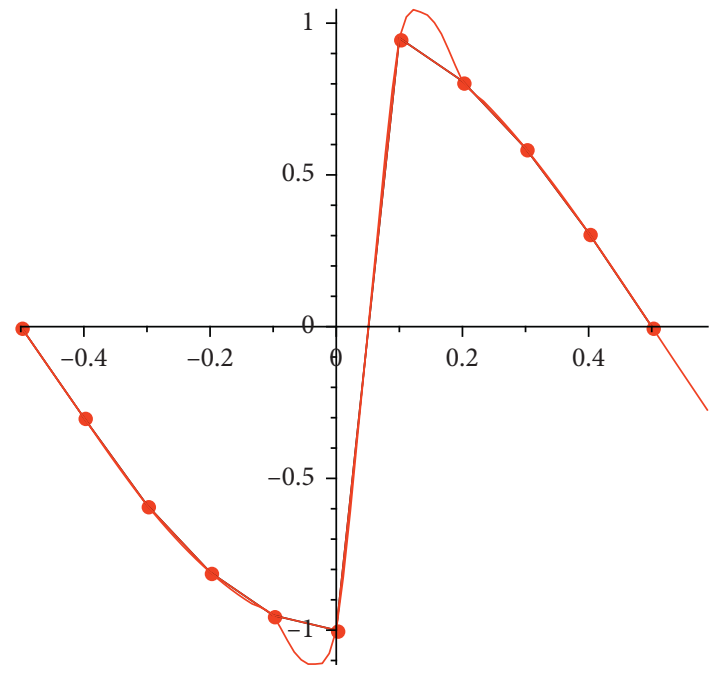

(a)

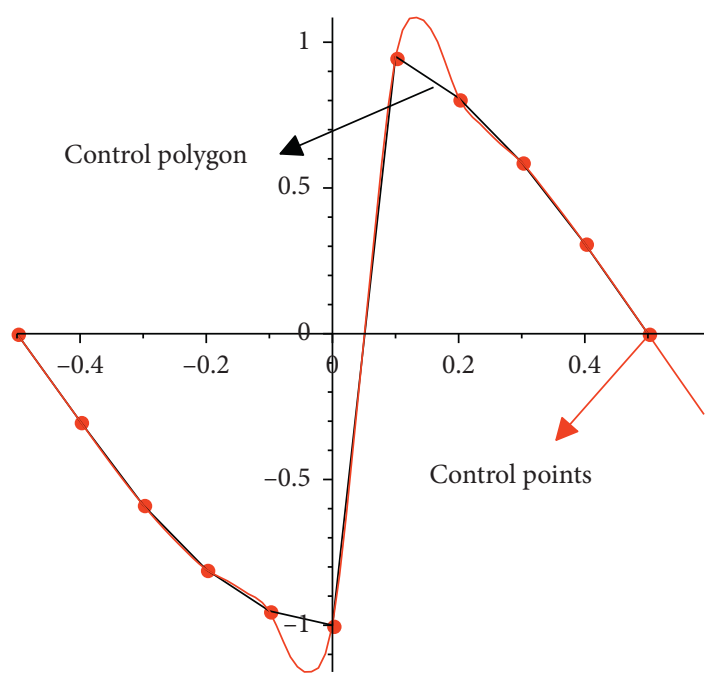

(c)

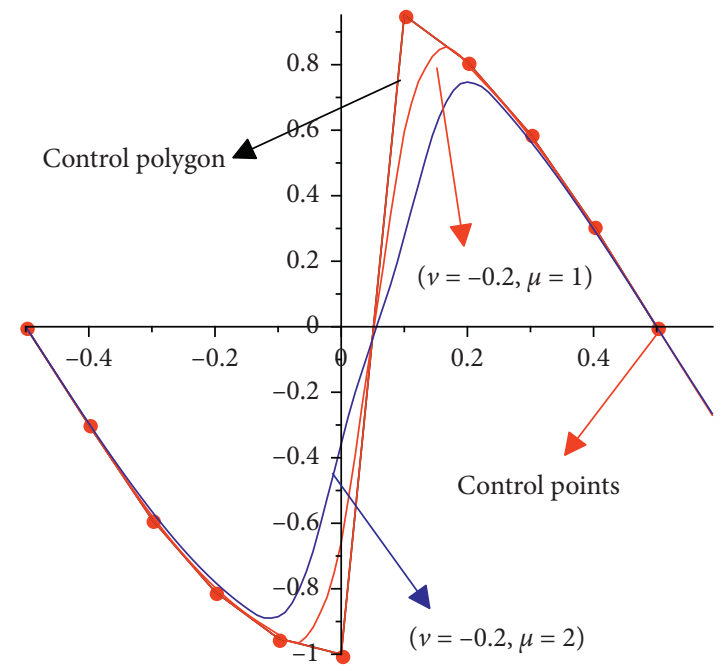

(b)

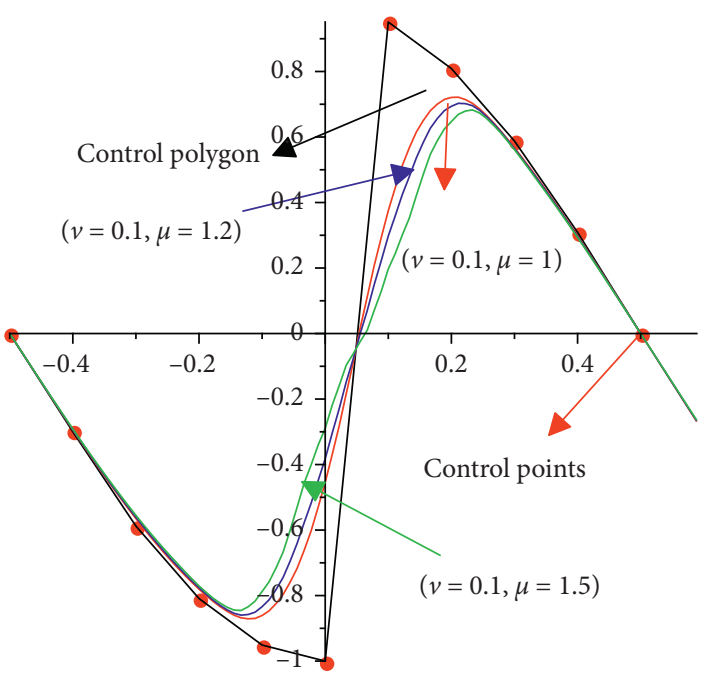

(d)

FIGURE 5: Red bullets are initial control points. Black solid lines are initial control polygons. Coloured lines show the fitted curves by our schemes in (b) and (d). 


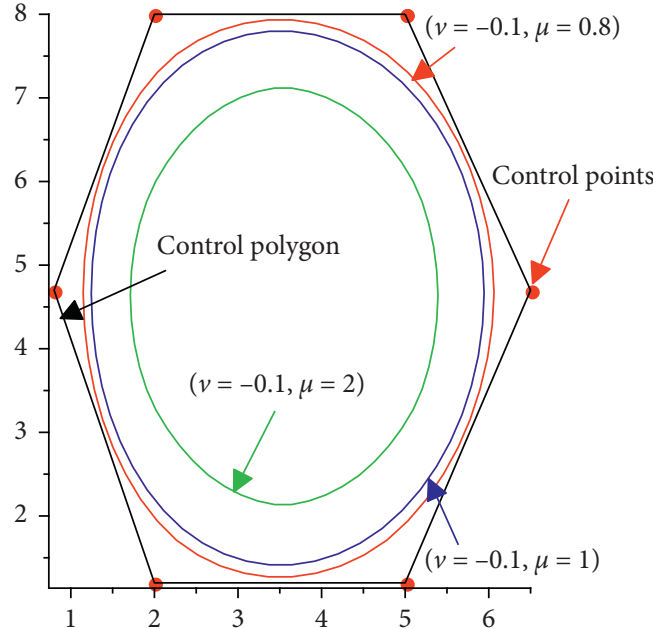

(a)

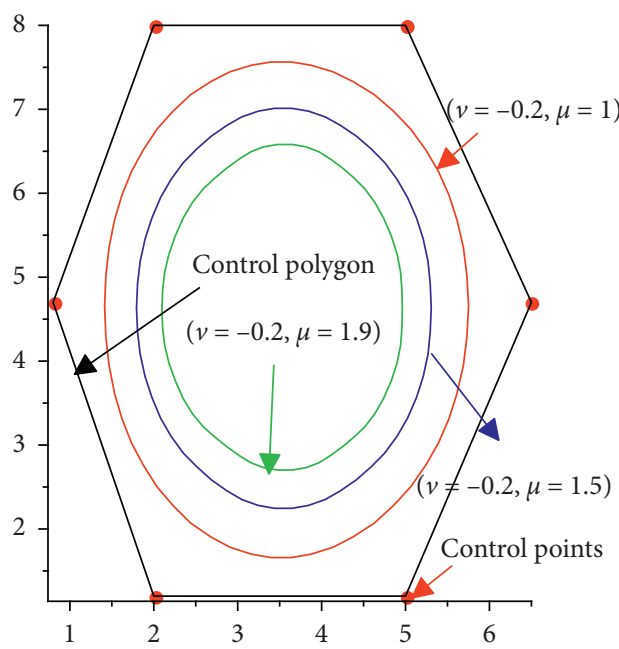

(c)

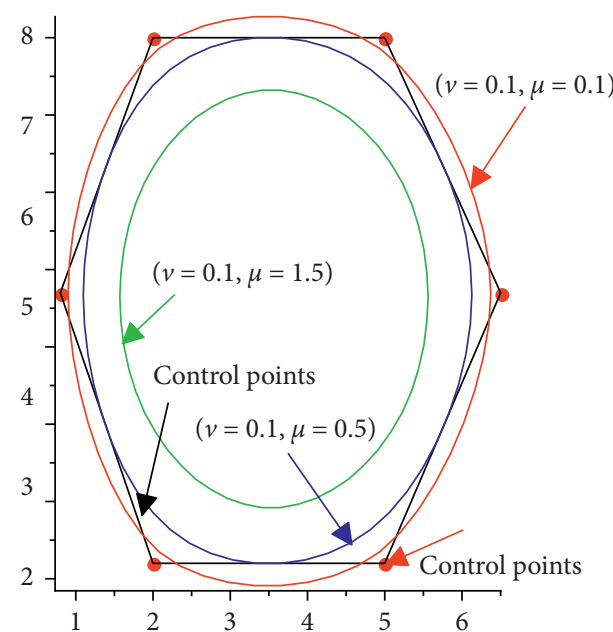

(b)

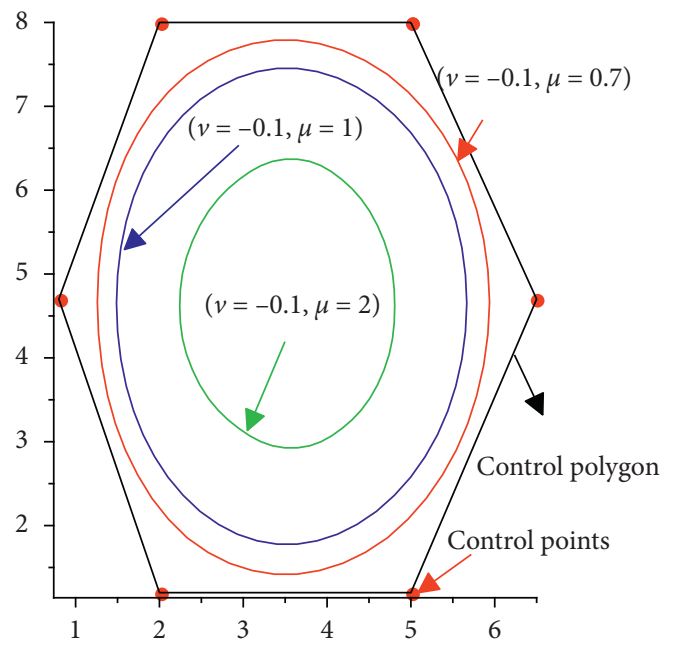

(d)

Figure 6: Red bullets show the initial control points. Black solid lines are the initial control polygon. Coloured lines show curves fitted by our subdivision scheme after two refinement steps. (a) 4-point combined scheme $\nu=-0.1, \mu=0.1,0.5,1.5$; (b) 4-point combined scheme $\nu=0.1, \mu=0.1,0.5,1.5$; (c) 6-point combined scheme $\nu=-0.1, \mu=1,1.5,1.9$; and (d) 6-point combined scheme $\nu=-0.3, \mu=1,1.3,1.5$.

\section{Geometrical Influence of $(\mathrm{CSS})_{a_{2 n+2}}$ in Curve Modeling}

In this section, we present models produced by the new combined 4-point and 6-point subdivision schemes. For modeling of $2 \mathrm{D}$ shapes by the combined schemes, we use the procedure which is explained in Figure 3 through a flowchart. This procedure is used in the following numerical examples for curve modeling.

Example 1. In this example, we draw the initial sketch $q^{0}$ by using the initial data $\left\{q_{i}^{0}: i \in \mathbb{Z}\right\}$. The initial sketch and the initial data are shown by black polygons and red bullets in Figure 4. Curves fitted by the subdivision schemes (CSS $)_{a_{4}}$ and (CSS) $a_{6}$ after four refinements steps for various values of $\mu$ and $\nu$ are shown in this figure. Figure 4(a) shows the curves fitted by a 4-point ternary combined scheme for $v=-0.2$ and $\mu=1,1.5,2$. Figure 4 (b) shows the curves fitted by a $4-$ point ternary combined scheme for $\nu=0.1$ and $\mu=-0.5,-0.8,1$. Figure 4 (c) shows the curves fitted by a $6-$ point ternary combined scheme for $v=-0.1$ and $\mu=0.8,1,1.5$. Figure $4(\mathrm{~d})$ shows the curves fitted by a 4 point ternary combined scheme for $\nu=0.1$ and $\mu=-0.2,-0.5,1$.

Example 2. In this experiment, we input the initial data taken from the discontinuous function:

$$
h(x)= \begin{cases}+\cos \pi x, & \text { if } x \in[0.5,0[ \\ -\cos \pi x, & \text { if } x \in[0,0.5] .\end{cases}
$$

Curves fitted by the first two members of our class of schemes are shown in Figures 5(b) and 5(d), respectively, and the curves fitted by the first two members of the CETISS 
are shown in Figures 5(a) and 5(c), respectively. We see that the CETISS produce Gibbs oscillations in the limit curves close to the discontinuity. It is also noted that our combined schemes do not produce Gibbs oscillations in the limit curves for certain values of the shape parameters.

Example 3. In this example, we take the initial data such that if we join this data by straight lines, we get a close polygonal shape. We smooth this shape by the first two members of our class of schemes and results are shown in Figure 6. This figure shows the flexible behaviour of our combined subdivision scheme. It also shows that shape parameters in the combined subdivision schemes provide grip on the limit curves.

\section{Conclusion}

In this research, we have constructed and analyzed a class of combined ternary subdivision schemes. The construction procedure is very simple and consists of only a few simple mathematical operations on the refinement rules of the two well-known classes of the schemes. But it gives us amazing results that are discussed theoretically and geometrically in detail. The class of schemes gives good smoothness and local control on the limit shapes. It also provides us a flexible environment to fit curves. The unnecessary oscillations in the limit curves of our combined schemes can be removed by changing the values of the shape parameters.

\section{Data Availability}

No datasets were generated or analyzsed during the current study.

\section{Conflicts of Interest}

The authors declare that they have no conflicts of interest.

\section{References}

[1] G. Mustafa, R. Hameed, D. Baleanu, and A. Mahmood, “A class of refinement schemes with two shape control parameters,” IEEE Access, vol. 8, no. 1, pp. 98316-98329, 2020.

[2] N. Dyn, D. Levin, and J. A. Gregory, "A 4-point interpolatory subdivision scheme for curve design," Computer Aided Geometric Design, vol. 4, no. 4, pp. 257-268, 1987.

[3] G. Deslauriers and S. Dubuc, "Symmetric iterative interpolation processes," Constructive Approximation, vol. 5, pp. 49-68, 1989.

[4] C. Beccari, G. Casciola, and L. Romani, "An interpolating 4point $C^{2}$ ternary non-stationary subdivision scheme with tension control," Computer Aided Geometric Design, vol. 24, no. 4, pp. 210-219, 2007.

[5] M. F. Hassan, I. P. Ivrissimitzis, N. A. Dodgson, and M. A. Sabin, "An interpolating 4-point $C^{2}$ ternary stationary subdivision scheme," Computer Aided Geometric Design, vol. 19, pp. 1-18, 2002.

[6] G. Mustafa, P. Ashraf, and J. Deng, "Generalized and unified families of interpolating subdivision schemes," Numerical Mathematics, vol. 7, no. 2, pp. 193-213, 2014.
[7] S. M. Hussain, A. Rehman, D. Baleanu, K. s. Nisar, A. Ghaffar, and S. A. A. Karim, "Generalized 5-point approximating subdivision scheme of varying arity," Mathematics, vol. 8, no. 474 , pp. 1-25, 2020.

[8] S. S. Siddiqi and M. Younis, "Construction of $m$-point binary approximating subdivision schemes," Applied Mathematics Letters, vol. 26, no. 3, pp. 337-343, 2013.

[9] R. Hameed and G. Mustafa, "Construction and analysis of binary subdivision schemes for curves and surfaces originated from Chaikin points," International Journal of Analysis, vol. 2016, Article ID 1092476, 15 pages, 2016.

[10] G. Mustafa and R. Hameed, "Families of univariate and bivariate subdivision schemes originated from quartic B-spline," Advances in Computational Mathematics, vol. 43, no. 5, pp. 1131-1161, 2017.

[11] O. Rioul, "Simple regularity criteria for subdivision schemes," SIAM Journal on Mathematical Analysis, vol. 23, no. 6, pp. 1544-1576, 1992.

[12] G. Mustafa, P. Ashraf, and J. Deng, "Generalized and unified families of interpolating subdivision schemes," Numerical Mathematics: Theory, Methods and Applications, vol. 7, no. 2, pp. 193-213, 2014.

[13] J. Pan, S. Lin, and X. Luo, "A combined approximating and interpolating ternary 4-point subdivision scheme," Applied Mathematics Letters, vol. 25, no. 12, pp. 2140-2146, 2012.

[14] D. Gottlieb and C. W. Shu, "On the Gibbs phenomenon and its resolution," SIAM Review, vol. 39, no. 4, pp. 644-668, 1997.

[15] S. Amat, J. Ruiz, J. C. Trillo, and D. F. Yanez, "Analysis of the Gibbs phenomenon in stationary subdivision schemes," Applied Mathematics Letters, vol. 76, pp. 157-163, 2018.

[16] K. Rehan and M. A. Sabri, "A combined ternary 4-point subdivision schemes," Journal of Computational and Applied Mathematics, vol. 276, pp. 278-283, 2016.

[17] N. Dyn, "Subdivision schemes in CAGD," in Advances in Numerical Analysis II: Wavelets, Subdivision Algorithms, and Radial Basis Functions, W. Light, Ed., Oxford University Press, Oxford, UK, 1992.

[18] F. Pitolli, "Ternary shape-preserving subdivision schemes," Mathematics and Computers in Simulation, pp. 185-194, 2014.

[19] P. Novara and L. Romani, "Complete characterization of the regions of C2 and C3 convergence of combined ternary 4point subdivision schemes," Applied Mathematics Letters, vol. 62, pp. 84-91, 2016. 